\title{
THE MEAN-FIELD QUANTUM HEISENBERG FERROMAGNET VIA REPRESENTATION THEORY
}

\author{
GIL ALON AND GADY KOZMA
}

\begin{abstract}
We use representation theory to write a formula for the magnetisation of the quantum Heisenberg ferromagnet. The core new result is a spectral decomposition of the function $\alpha_{k} 2^{\alpha_{1}+\cdots+\alpha_{n}}$ where $\alpha_{k}$ is the number of cycles of length $k$ of a permutation. In the mean-field case, we simplify the formula further, arriving at a closed-form expression for the magnetisation, which allows to analyse the phase transition.
\end{abstract}

\section{INTRODUCTION}

The quantum Heisenberg ferromagnet is one of the simplest multiparticle quantum system one may imagine. Particles do not move, they are fixed in lattice positions. Each particle is endowed with a spin and the interactions are spin interactions. Since spins are described by $2 \times 2$ matrices the entire system may be described by a $2^{n} \times 2^{n}$ matrix, removing the need to involve operator theory, as is common in quantum mechanics.

In a landmark paper, Dyson, Lieb and Simon analysed the quantum Heisenberg antiferromagnet using an approach known as quantum reflection positivity [17]. Surprisingly, the ferromagnetic version could not be analysed using the same approach and is still open (the classical version of reflection positivity is less sensitive to model details, see $[21,19,20])$.

An initially unrelated line of research, inspired by classical physics, was that of particle systems. In 1970, Spitzer [30] defined the exclusion process: given a graph $G$ (Spitzer was mainly interested in the case that $G$ is $\mathbb{Z}^{d}$ ) put particles on its vertices, coloured white and black, and Poisson clocks on the edges. When a clock rings, the two particles on the sides of that edge are exchanged. A similar process, when the particles do not have two types but rather each particle is unique is called the interchange process. In 1981 Diaconis and Shahshahani [16], motivated by problems in card shuffling, proved that the mixing time of the interchange process on the complete graph on $n$ vertices is $\frac{1}{2} \log n$. In physics' parlance, studying the complete graph is called mean-field, so one may say that Diaconis and Shahshahani studied the mean-field interchange process.

The connection between the quantum Heisenberg ferromagnet and the interchange process was first derived using physics arguments in [27], and then made rigorous by works of Conlon and Solovej [11] and Tóth [35]. The inverse temperature of the quantum system translates to the time the interchange process is run, and various physical quantities translate to various questions about the cycle structure of the permutation of the interchange particles (one such quantity will be described in details below). Interestingly, the most physically relevant setting, that 
of fixed temperature and system size going to infinity, translates to studying the interchange process at constant time, in particular before its mixing time.

This connection generated a lot of interest since the interchange process is subject to many different attack vectors, both probabilistic and algebraic. A notable result achieved by a purely probabilistic approach is that of Schramm [29], who did a very fine analysis of the cycle structure of the interchange process in the mean-field case. His approach was recently applied to the hypercube [25] and to the Hamming graph [1]. The case that $G$ is a tree was studied by Angel [5] and Hammond [22, 23]. In particular, [23] shows a sharp phase transition (when the tree has sufficiently high degrees), an impressive result given that the system has no inherent monotonicity.

The algebraic approach starts from the observation that the interchange process is a random walk on $\mathrm{Sym}_{n}$, the group of permutations of $n$ elements. Hence representation theory may be applied to it, somewhat similarly to the classic application of Fourier transform to studying simple random walk. This is most effective in the mean-field case, since then representation theory gives full linearisation of the problem. This fact is behind the work of Diaconis and Shahshahani. It also allows to study the interchange process at constant time, see [7]. Nevertheless, representation theory can also be used for general graphs. For example, it gives a simple formula for the probability that the interchange process is one large cycle, see [3].

In the discussion so far we have ignored an important issue of weighting that appears when translating from the quantum Heisenberg ferromagnet to the interchange process. This weighting means that all the results for the interchange process that we have quoted do not translate directly to the quantum model, and may serve only as a heuristic guide. To explain this any further we need to delve into the details of the interchange process, and we will do so now. (The quantum system will be defined in details later, in $\$ 1.4$ )

1.1. The interchange process. Let us now define the interchange process (a.k.a. the stirring process) formally, directly defining it as a random walk on $\mathrm{Sym}_{n}$. We start with the walker at the identity permutation and run it in continuous time. Every edge of the interaction graph $G$ is attached to a Poisson clock of rate 1, and when the clock corresponding to an edge $(i, j)$ rings, the position of the walker at that time $\pi(t)$ is changed to $(i j) \pi(t)$ i.e. a transposition is composed from the left. Notice the attractive feature of using continuous time: for every vertex $i$, $\pi(t)(i)$ is a continuous-time random walk on $G$. Readers familiar with the discretetime formulation of the interchange process should note that time $t$ corresponds to approximately $t n$ steps of the discrete time process.

Next, let us define two physical quantities, the free energy $Z$ and the expected squared magnetisation $m$, already translated (via [35]) to the interchange process. For a permutation $\pi$, denote by $\alpha_{k}(\pi)$ the number of cycles of length $k$ in $\pi$ and by $\alpha(\pi)=\sum_{i} \alpha_{i}(\pi)$ the total number of cycles in $\pi$. Define

$$
Z(t)=\mathbb{E}\left(2^{\alpha(\pi(t))}\right) \quad m^{2}(t)=\frac{1}{Z(t)} \mathbb{E}\left(\left(\sum_{k} k^{2} \alpha_{k}(\pi(t)) 2^{\alpha(\pi(t))}\right) .\right.
$$

In $\S 1.4$ we will explain the connection of $Z$ and $m$ to the quantum system, but for now (1) will suffice as the definition.

The most important conjecture about the quantum Heisenberg ferromagnet is that in dimension $d \geq 3$ there is a phase transition in the behaviour of the magnetisation. Let us state formally a version of it: 
Conjecture. For every $d \geq 3$ there is a critical value, denoted by $t_{c}$, with the following property. Let the interaction graph $G$ be $\{1, \ldots, l\}^{d}$ where $\{1, \ldots, l\}$ is given the graph structure of the cycle and the power is the usual ("square") graph product. Then

$$
\begin{aligned}
& t<t_{c} \Longrightarrow m(t) \lesssim \sqrt{n} \\
& t>t_{c} \Longrightarrow m(t) \gtrsim n
\end{aligned}
$$

(since $n$ denotes for us the size of the graph, we have $n=l^{d}$ in the conjecture). Here and below, the notation $X \lesssim Y$ means that there is some constant $C>0$, which may depend on $d$ and on $t$, but not on $n$, such that $X \leq C Y$ (the conjecture is as $n$ (or $l$ ) goes to $\infty$ ). It is easy to show that for $t$ sufficiently small the behaviour is indeed as stated i.e. $m(t) \lesssim \sqrt{n}$. What is not known is that there exists an ordered phase at all i.e. that for some $t$ sufficiently large we have $m(t) \gtrsim n$.

The factors $2^{\alpha}$ that appear in (1) are the weights that we mentioned in the previous section. The result of Tóth from [35] is that the naturally defined magnetisation translates precisely to the quantity $m$ from (1). But from the point of view of the interchange process, it is natural to simply ask if $\sum k^{2} \alpha_{k}$ undergoes a phase transition in the time $t$. And indeed, all results we mentioned in the previous section are of this form. For example, Berestycki and Durrett [6] show that, for $G$ being the complete graph, there is a $t_{c}$ such that

$$
t<\frac{t_{c}}{n} \Longrightarrow \mathbb{E}\left(\sum k^{2} \alpha_{k}(\pi(t))\right) \lesssim n \quad t>\frac{t_{c}}{n} \Longrightarrow \frac{1}{n} \mathbb{E}\left(\sum k^{2} \alpha_{k}(\pi(t))\right) \rightarrow \infty
$$

(we compare $t$ to $t_{c} / n$ rather than to $t_{c}$ because of the high degree of the complete graph, which speeds the process $n$-fold). Schramm [29] improved this by showing that at $t>t_{c} / n, \mathbb{E}\left(\sum k^{2} \alpha_{k}(\pi(t))\right) \gtrsim n^{2}$ (both results give more information than just the expectation, for example Schramm gave a description of the distribution of the sizes of the large cycles). Despite all this progress, the case where the interaction graph $\mathrm{G}$ is $\{1, \ldots, l\}^{d}$ is still open, even for the question without the weights.

Our purpose in this paper is twofold:

(i) Prepare for an algebro-analytic attack on the non mean-field case.

(ii) Give an alternative and potentially more precise analysis of the mean-field case.

We start with the mean-field results, as they do not require representation theory to state. Our thoughts about how the non mean-field case may be attacked via these results are best discussed after some preliminaries and we will do so in §1.2.1. As above, the high degree requires a scaling of the time:

Theorem 1. When the interaction graph is the complete graph we have

$$
\begin{aligned}
& t<\frac{2}{n} \Longrightarrow m(t) \lesssim \sqrt{n} \\
& t>\frac{2}{n} \Longrightarrow m(t) \gtrsim n .
\end{aligned}
$$

This theorem is not new, it can be inferred from a different paper of Tóth, [34], which attacks the mean-field quantum problem using an approach not related to the interchange process ([34] discusses the free energy, but the magnetisation should follow from it by differentiation). Penrose gave a second proof at about the same time [26]. Another proof of theorem 1 was given recently in $[9,10]$, valid when the 
term $2^{\alpha}$ in (1) is replaced by $\theta^{\alpha}$ for an arbitrary $\theta>0$, using the techniques of Schramm [29]. However, our techniques give a closed formula for the magnetisation:

Theorem 2. Under the same conditions as theorem 1,

$$
m_{n}^{2}(t)=n \frac{\sum_{b, k} k \psi(b, k, t)}{\sum_{b, k} \psi(b, k, t)}
$$

the sums running over $k$ from 1 to $n$ and $b$ from 0 to $\lfloor(n-k) / 2\rfloor$, and where $\psi$ is defined, for $b>0$ by

$$
\begin{aligned}
\psi(b, k, t)=(a+1 & -b)\left(\begin{array}{c}
n \\
k-1, a+1, b
\end{array}\right) \cdot e^{-t(a b+b)}\left[\frac{(a+1-b) y^{a+b+2}(1-y)^{k-1}}{k}\right. \\
& +y^{b}(a+1-b+k) \int_{y}^{1} x^{a+1}(1-x)^{k-1} d x \\
& \left.+y^{a+1}(a+1-b-k) \int_{y}^{1} x^{b}(1-x)^{k-1} d x\right]
\end{aligned}
$$

and for $b=0$ by

$$
\psi(0, k, t)=(a+1)\left(\begin{array}{l}
n \\
k
\end{array}\right)\left[y^{n-k+1}(1-y)^{k-1}+k \int_{y}^{1} x^{n-k}(1-x)^{k-1} d x\right]
$$

and where $a=n-k-b$ and $y=e^{-t k}$.

Concluding theorem 1 from theorem 2 is an exercise and we do it in the end. Certainly, a finer analysis of theorem 2 would give a very detailed picture of the phase transition, but we prefer to stick with the relatively rough theorem 1 as our focus here is the proof of theorem 2. The proof uses representation theory, so let us turn to this topic now.

1.2. Representations of the symmetric group. Group representations are a non-commutative analogue of the Fourier transform, so let us use notation suggestive of it. Let $f: \operatorname{Sym}_{n} \rightarrow \mathbb{C}$ be some function and let $R: \operatorname{Sym}_{n} \rightarrow \operatorname{GL}\left(V_{R}\right)$ be some representation of $\mathrm{Sym}_{n}$. We denote

$$
\widehat{f}(R)=\sum_{\pi \in S_{n}} f(\pi) R(\pi)
$$

so $\widehat{f}(R)$ is a linear map from $V_{R}$ to $V_{R}$. We have an analogue of Parseval's formula (see, e.g., [14, theorem 4.1]),

$$
\langle f, g\rangle=\sum_{R} \frac{d_{R}}{n !} \operatorname{Tr}\left(\widehat{f}(R) \widehat{g}(R)^{*}\right)
$$

where the sum is over all (equivalence classes of) irreducible unitary representations of $\operatorname{Sym}_{n} ; d_{R}=\operatorname{dim} V_{R}$ i.e. the dimension of the space the representation $R$ acts upon; and $\langle f, g\rangle=\sum_{\pi \in \operatorname{Sym}_{n}} f(\pi) g(\pi)$ i.e. is not normalised. To apply this to, for example, the partition function, $Z(t)=\mathbb{E}\left(2^{\alpha(\pi(t))}\right)$ denote by $p_{t}(\pi)$ the probability that $\pi(t)=\pi$ and then

$$
Z(t)=\left\langle 2^{\alpha}, p_{t}\right\rangle=\sum_{R} \frac{d_{R}}{n !} \operatorname{Tr}\left(\widehat{2^{\alpha}}(R) \widehat{p}_{t}(R)^{*}\right) .
$$


Now, $2^{\alpha}$ is a class function i.e. a function which depends only on the cycle structure of the permutation. Hence Schur's lemma tells us that $\widehat{2^{\alpha}}(R)$ is a scalar matrix for any irreducible $R$, though it does not give the value of the scalar. As for $p_{t}$, it is a class function only in the mean-field case. This is what makes the mean-field case amenable to analysis, and in fact, $\widehat{p_{t}}$ was calculated explicitly by Diaconis and Shahshahani [16].

Thus theorem 2 will follow once we calculate $\widehat{2^{\alpha}}$ (for $Z$ ) and $\widehat{\alpha_{k} 2^{\alpha}}$ (for $m$ ). To state these, recall that the irreducible representations of $S_{n}$ can be indexed by partitions of $n$ (see e.g. [24]). We denote by $R_{\lambda}$ the irreducible representation of $S_{n}$ corresponding to the partition $\lambda$. We use the standard notation $a^{b}$ for a repetition in a partition, so, for example, $\left[3,1^{3}\right]$ is the partition $6=3+1+1+1$. The case $\widehat{2^{\alpha}}$ is not difficult (we give the details below):

$$
\widehat{2^{\alpha}}(\lambda)=\frac{n !}{d_{\lambda}} I_{\lambda} \begin{cases}a-b+1 & \lambda=[a, b] \\ 0 & \text { otherwise }\end{cases}
$$

where $I_{\lambda}$ is the identity matrix of the vector space $V_{\lambda}$ (recall that the values of the non-commutative Fourier transform are matrices). Here and below we use the short-hand notation $V_{\lambda}=V_{R_{\lambda}}, d_{\lambda}=d_{R_{\lambda}}$ and $\widehat{f}(\lambda)=\widehat{f}\left(R_{\lambda}\right)$.

For the function $\alpha_{k} 2^{\alpha}$ we need to introduce some further notation. Recall that a skew diagram is the set difference $\lambda \backslash \mu$ of two Young diagrams $\lambda, \mu$, and a border strip is a connected skew diagram which does not contain a $2 \times 2$ square. When we say about a skew diagram that it is connected we mean connection through edges, so $\Phi \backslash \square=\square$ is not considered to be connected. For a skew diagram $\mu$, the height $\operatorname{ht}(\mu)$ is defined to be the number of its rows.

Theorem 3. We have $\widehat{\alpha_{k} 2^{\alpha}}(\lambda)=\frac{n !}{d_{\lambda}} a_{\lambda, k} I_{\lambda}$ where

$$
a_{\lambda, k}=\frac{2}{k} \begin{cases}(a-b+1) \cdot(-1)^{\mathrm{ht}(\lambda \backslash[a, b])+1} & \exists[a, b] \dashv n-k \text { such that } \\ 0 & \lambda \backslash[a, b] \text { is a border strip } \\ & \text { otherwise. }\end{cases}
$$

It is easy to check that $a$ and $b$ are uniquely defined by $\lambda$ and $k$, if they exist. Of course, the notation $[a, b] \dashv n-k$ i.e. $[a, b]$ is a partition of $n-k$, is nothing but $a \geq b \geq 0$ and $a+b=n-k$. We note as a corollary of theorem 3 that the Fourier transform is supported on diagrams of the form $\left[a, b, c, 1^{d}\right]$.

Let us sketch how theorem 2 follows from theorem 3. Define the laplacian $\Delta$ : $S_{n} \rightarrow \mathbb{R}$ by

$$
\Delta=\sum_{i \sim j} \nabla_{i j} \quad \nabla_{i j}(\pi)= \begin{cases}1 & \pi=\mathrm{id} \\ -1 & \pi=(i j) \\ 0 & \text { otherwise }\end{cases}
$$

where id is the identity permutation; and where $i \sim j$ means that $i$ and $j$ are neighbours in the interaction graph $G$. Then $p_{t}=e^{-t \Delta}$ where the exponentiation is in the convolution algebra over $\operatorname{Sym}_{n}$ (or in the group ring $\mathbb{R}\left[\mathrm{Sym}_{n}\right]$, which is the same). Since Fourier transform translates convolution into product we also get $\widehat{p}_{t}(\lambda)=e^{-t \widehat{\Delta}(\lambda)}$ where this time this is simply exponentiation of matrices. By $(2)$ we get

$$
Z \stackrel{(2)}{=} \sum_{R} \frac{d_{R}}{n !} \operatorname{Tr}\left(\widehat{2^{\alpha}}(R) \widehat{p_{t}}(R)^{*}\right) \stackrel{(3)}{=} \sum_{b=0}^{n / 2}(n-2 b+1) \operatorname{Tr}\left(\widehat{p_{t}}([n-b, b])\right) .
$$


Denote by $\rho_{1}(\lambda), \ldots, \rho_{d_{\lambda}}(\lambda)$ the eigenvalues of $\widehat{\Delta}(\lambda)$ and get

$$
\begin{aligned}
Z & =\sum_{b=0}^{n / 2}(n-2 b+1) \operatorname{Tr}(\exp (-t \widehat{\Delta}([n-b, b]))) \\
& =\sum_{b=0}^{n / 2}(n-2 b+1) \sum_{i=1}^{d_{[n-b, b]}} \exp \left(-t \rho_{i}([n-b, b])\right) .
\end{aligned}
$$

A similar formula holds for $m$,

$$
m^{2}=\frac{1}{Z} \sum_{k=1}^{n} k^{2} \sum_{\lambda \dashv n} a_{\lambda, k} \sum_{i=1}^{d_{\lambda}} \exp \left(-t \rho_{i}(\lambda)\right)
$$

where $a_{\lambda, k}$ are given by theorem 3 .

The calculation so far was for any $G$. We now assume $G$ is the complete graph. In this case, $\Delta$ is in the center of the group ring of $\operatorname{Sym}_{n}$, so by Schur's lemma, $\rho_{1}(\lambda)=\rho_{2}(\lambda)=\cdots=\rho_{d_{\lambda}}(\lambda)$, and we simplify our formulas writing

$$
Z=\sum_{b=0}^{n / 2} d_{[n-b, b]} \rho([n-b, b]) \quad m=\sum_{k} k^{2} \sum_{\lambda} a_{\lambda, k} d_{\lambda} \exp (-t \rho(\lambda))
$$

where $\rho(\lambda)$ denotes the common value. Next, $\rho(\lambda)$ can be calculated by examining the trace of $\Delta$, leading to

$$
\rho(\lambda)=\left(\begin{array}{l}
n \\
2
\end{array}\right)\left(1-\frac{\chi_{\lambda}((12))}{d_{\lambda}}\right)
$$

where $\chi_{\lambda}$ is the character of $R_{\lambda}$ i.e. $\chi_{\lambda}(\sigma)=\operatorname{Tr} R_{\lambda}(\sigma)$. The value $\frac{\chi_{\lambda}((12))}{d_{\lambda}}$ is called the character ratio and has a formula, mainly due to Frobenius, which is quoted in [16]. Thus we get explicit formulas for $Z$ and $m$.

1.2.1. We end this part of the introduction with a few remarks on the case where the interaction graph is not the complete graph. Here the main difficulty is to learn something about $\widehat{p_{t}}$, or equivalently about $\widehat{\Delta}$. Since we eventually are only interested in the trace of $e^{-t \widehat{\Delta}}$, we see that we need to understand the eigenvalues of $\widehat{\Delta}(\lambda)$, and not for any $\lambda$, but just for $\lambda$ where the coefficients in theorem 3 are non-zero, i.e. for $\lambda$ of the form $\left[a, b, c, 1^{d}\right]$. Denote the eigenvalues which correspond to a given graph $G$ and diagram $\lambda$ by $\rho_{i}(\lambda ; G)$, ordered in increasing order.

Let us mention two interesting bounds on these eigenvalues. The first is a bound for the minimum eigenvalue,

$$
\rho_{1}\left(\lambda ;\{1, \ldots, l\}^{d}\right) \gtrsim \frac{1}{n l^{2}} \rho_{1}\left(\lambda ; K_{n}\right) .
$$

(Of course, $\rho\left(\lambda ; K_{n}\right)$ is given by (5)). This follows from a comparison argument (an operator version of the classic multi-commodity flow argument). See [15],[12, $\S 5$ ] or [4], and see also [31] for more about the minimal eigenvalue. (In [4] we demonstrate an example where the information about the minimal eigenvalue is enough to prove the existence of a phase transition.) Probably, the estimate (6) is tight up to the value of the constant. A second fact, known only for two-rows Young diagrams, is that the statistics of the $\rho_{i}([n-k, k])$ is the same as that of sums of $k$-tuples of $\rho_{i}([n-1,1])$, which are simply the eigenvalues of the Laplacian on the interaction graph $G$ [36]. Thus there is good control of two scales, the smallest and 
the largest, and what is missing to prove the conjecture is control of the eigenvalues in intermediate scales.

1.3. The Frobenius map. We will now delve a little deeper into the algebraic aspects of this work, namely the proof of theorem 3. Before starting, let us make a remark on one natural approach to prove theorem 3 which we could not make work. Recall that we wish to calculate $\widehat{\alpha_{k} 2^{\alpha}}$ or, alternatively, to present $\alpha_{k} 2^{\alpha}$ as a combination of irreducible characters. As already mentioned, $\widehat{2^{\alpha}}$ is supported on Young diagrams of the form $[a, b](3)$. Moreover, $\widehat{\alpha_{k}}$, as shown in [3], is supported on Young diagrams of the form $\left[a, b, 1^{c}\right]$. It may seem natural to try and deduce $\widehat{\alpha_{k} 2^{\alpha}}$ directly from these decompositions. However, the problem of writing the product (also known as the Kronecker product) of two irreducible characters as a linear combination of irreducible characters is highly nontrivial: It has been studied for more than eighty years. Many partial results are known, but not one suitable for this case. In [28] Rosas found the decomposition of a Kronecker product for a two-row diagram with both a hook-shaped diagram (i.e. a diagram of the form $\left.\left[a, 1^{b}\right]\right)$ and with a second two-row diagram. This falls short of the case in question. Our proof thus takes a different route, which we now sketch.

As in [3], we express the decomposition of $\widehat{\alpha_{k} 2^{\alpha}}$ as a sum of irreducible characters via the Frobenius characteristic map ch. Let us recall this classical object. The Frobenius map is a function from the space of class functions on $\mathrm{Sym}_{n}$ to the ring of symmetric functions, which maps $\chi_{\lambda}$, the character of the representation $\lambda$, to the Schur polynomial $S_{\lambda}$. See [33, Definition 7.10.1 or $\left.\S \S 7.10,7.15\right]$ for the Schur polynomials. We will prove that

$$
\operatorname{ch}\left(\alpha_{k} 2^{\alpha}\right)=u_{k} \cdot \operatorname{ch}\left(2^{\alpha}\right)
$$

where $u_{k}=\sum_{i} x_{i}^{k}$. Consequently, the decomposition of $u_{k} \operatorname{ch}\left(2^{\alpha}\right)$ to Schur polynomials can be obtained from that of $\operatorname{ch}\left(2^{\alpha}\right)$ by a variant of the Murnaghan-Nakayama rule. Theorem 3 follows as a consequence.

Another point of the calculation we wish to stress is the use of hook numbers. The hook numbers of a partition $n \vdash \lambda$ are defined by

$$
\lambda^{(i)}=\lambda_{i}+\mathrm{ht}(\lambda)-i .
$$

It turns out that all of $a_{\lambda}, d_{\lambda}$ and, in the mean-field case, $\rho(\lambda)$ can be conveniently expressed in terms of the hook numbers of $\lambda$. This change of variables yields a considerable simplification of the expression for $\mathbb{E}\left(\alpha_{k}(\pi(t)) 2^{\alpha(\pi(t))}\right)$. We will evaluate the sums in this expression (in a similar way to the calculation in [7]), and obtain a closed form expression in terms of some incomplete Beta integrals.

1.4. The quantum model. We now return to the starting point of this paper, the quantum Heisenberg ferromagnet, and describe it in detail. We are given $n$ interacting particles. The interaction scheme is defined by a graph $G$ on $n$ vertices, and two particles interact if there is an edge between the corresponding vertices. Each particle is spin- $\frac{1}{2}$ and interactions are spin interactions. Hence each particle corresponds to a vector in $\mathbb{C}^{2}$ and the state space of the entire system is $\otimes_{i=1}^{n} \mathbb{C}^{2}$. Recall the Pauli matrices $\sigma^{x}=\left(\begin{array}{ll}0 & 1 \\ 1 & 0\end{array}\right), \sigma^{y}=\left(\begin{array}{cc}0 & -i \\ i & 0\end{array}\right)$ and $\sigma^{z}=\left(\begin{array}{cc}1 & 0 \\ 0 & -1\end{array}\right)$ and define the Pauli matrix at particle $i$ by

$$
\sigma_{i}^{x}=\underbrace{I \otimes \cdots \otimes I}_{i-1 \text { times }} \otimes \sigma^{x} \otimes \underbrace{I \otimes \cdots \otimes I}_{n-i \text { times }}
$$


and ditto for $\sigma^{y}$ and $\sigma^{z}$. The Hamiltonian is now

$$
H=-\frac{1}{4} \sum_{i \sim j} \sigma_{i}^{x} \sigma_{j}^{x}+\sigma_{i}^{y} \sigma_{j}^{y}+\sigma_{i}^{z} \sigma_{j}^{z}
$$

where the notation $i \sim j$ means that $i$ and $j$ are neighbours in the interaction graph $G$. The partition function at temperature $T$ is

$$
Z=Z_{n}(\beta)=\operatorname{Tr} \exp (-\beta H)
$$

where $\beta=1 / T$. The expected square magnetisation is

$$
m_{n}^{2}(\beta)=\frac{1}{Z_{n}(\beta)} \operatorname{Tr}\left(\exp (-\beta H)\left(\sum_{i} \sigma_{i}^{z}\right)^{2}\right) .
$$

The result of [35] imply that the $m$ defined above is exactly the one defined in (1), with $t=\frac{1}{2} \beta$, i.e. the inverse temperature in the quantum model becomes the time in the interchange model (the quantum and interchange definitions of $Z$ differ by a constant).

This might be a good point to indicate a small issue regarding the definition of magnetisation. The magnetisation as defined in [35] is the residual magnetisation, i.e., an external field $h$ is applied (mathematically, the term $h \sum \sigma_{i}^{z}$ is added to the Hamiltonian), the magnetisation is calculated as a function of $h, n$ is taken to infinity and then $h$ is taken to 0 . This residual magnetisation (denote it by $m^{*}$ ) can also be described by the interchange process,

$$
m^{*}(\beta)=\frac{1}{2} \lim _{M \rightarrow \infty} \lim _{n \rightarrow \infty} \frac{1}{Z_{n}(\beta)} \sum_{k=M+1}^{n} \frac{k}{n} \mathbb{E}\left(\alpha_{k} 2^{\alpha(\pi(\beta))}\right)
$$

assuming the limits exist, see [35, (5.2)]. (unfortunately, while the formula (8) for $m^{*}$ can be found in [35] explicitly, the equivalent formula for $m$, i.e. the equivalence of our two definitions for $m,(1)$ and in (7) is not written as such in [35]. Nevertheless the argument is sufficiently similar to the arguments of [35] that we feel we may omit it).

It is easy to see that $m(t) \gtrsim n$ as $n \rightarrow \infty$ implies that $m^{*}(t)>0$, since the first says that there are cycles of linear size, while the latter says that there is some "mass" in cycles of size larger than constant. As this is quite standard, we postpone the proof to the appendix.

1.5. Recap of notation. Throughout this paper, we will analyse the continuous time interchange process on $n$ particles, with respect to the complete graph. See [3] for more details on the interchange process. We denote by $\pi(t)$ the permutation at time $t \geq 0$.

For a permutation $\pi \in S_{n}$, we denote by $\alpha_{k}(\pi)$ the number of cycles of length $k$ in $\pi$, and by $\alpha(\pi)=\sum_{i} \alpha_{i}(\pi)$ the total number of cycles in $\pi$. We will denote by $c_{i}(\pi)$ the size of the cycle of $\pi$ containing $i$.

For a partition $n \vdash \lambda$, we denote by $R_{\lambda}$ the irreducible representation of $\operatorname{Sym}_{n}$ associated with $\lambda$, by $d_{\lambda}$ its dimension, and by $\chi_{\lambda}$ the corresponding irreducible character. We denote by $a_{\lambda, k}$ the numbers from theorem 3 . When $k$ is clear from context, we will often remove it, writing simply $a_{\lambda}$.

We let ch be the Frobenius characteristic map. It is a function from the space of class function on $\mathrm{Sym}_{n}$ to the ring of symmetric functions, which maps $\chi_{\lambda}$ to the Schur polynomial $S_{\lambda}$. 


\section{THE CHARACTER DECOMPOSITION}

The main step in the proof of theorem 3 is the calculation of $\operatorname{ch}\left(\alpha_{k} 2^{\alpha}\right)$. We state it as a result.

Theorem 4. We have

(i)

$$
\operatorname{ch}\left(2^{\alpha}\right)=\sum_{n \vdash[a, b]}(a-b+1) S_{[a, b]}
$$

where $b$ is allowed to be 0 , in which case $[a, b]$ means $[a]$. In other words, we sum over all couples $a, b$ such that $a \geq b \geq 0$ and $a+b=n$.

(ii) For $1 \leq k \leq n$,

$$
\operatorname{ch}\left(\alpha_{k} 2^{\alpha}\right)=\frac{2}{k} u_{k} \sum_{n-k \vdash[a, b]}(a-b+1) S_{[a, b]}
$$

where $u_{k}=\sum_{i} x_{i}^{k}$.

Proof of (i). The polynomial $\sum_{\pi \in S_{n}} x^{\alpha(\pi)}$ is the generating function of the Stirling numbers of the first kind, and is equal to $\prod_{i=0}^{n-1}(x+i)$ (see [32, proposition 1.3.4]). In particular, we have $\sum_{\pi \in S_{n}} 2^{\alpha(\pi)}=(n+1)$ !. For any $n \vdash \lambda=\left[\lambda_{1}, \lambda_{2}, \ldots, \lambda_{r}\right]$ (with $\lambda_{1} \geq \cdots \geq \lambda_{r}>0$ ), consider the corresponding partition of $\{1,2, \ldots, n\}$ to sets $A_{\lambda}^{i}$ of sizes $\lambda_{1}, \ldots, \lambda_{r}$ :

$$
A_{\lambda}^{i}=\mathbb{Z} \cap\left(\sum_{j=1}^{i-1} \lambda_{j}, \sum_{j=1}^{i} \lambda_{j}\right]
$$

and let $T_{\lambda} \cong \prod \operatorname{Sym}_{\lambda_{i}}$ be the group of permutations in $\mathrm{Sym}_{n}$ preserving all the sets $A_{\lambda}^{i}$. Let $M_{\lambda}$ be the sum of all monomials which can be obtained from $\prod x_{i}^{\lambda_{i}}$ by a permutation of the variables $\left\{x_{i}\right\}$. We will use the following formula from [3, lemma 2] (valid for any class function $f$ on $\mathrm{Sym}_{n}$ ):

$$
\operatorname{ch}(f)=\sum_{n \vdash \lambda}\left(\frac{1}{\left|T_{\lambda}\right|} \sum_{\pi \in T_{\lambda}} f(\pi)\right) M_{\lambda}
$$

It follows that

$$
\operatorname{ch}\left(2^{\alpha}\right)=\sum_{n \vdash \lambda} M_{\lambda} \prod_{i}\left(\lambda_{i}+1\right)
$$

Clause (i) will thus be proved once we show that

$$
\sum_{n \vdash \lambda} M_{\lambda} \prod_{i}\left(\lambda_{i}+1\right)=\sum_{n \vdash[a, b]}(a-b+1) S_{[a, b]}
$$

To see (10) we first claim that

$$
\sum_{n \vdash \lambda} M_{\lambda} \prod_{i}\left(\lambda_{i}+1\right)=\sum_{i=0}^{n} S_{[i]} S_{[n-i]}
$$

Indeed, this is proved by comparing the coefficient of $\prod x_{i}^{\lambda_{i}}$ in both sides: It is clearly $\prod\left(\lambda_{i}+1\right)$ on the left hand side, and it is also $\prod\left(\lambda_{i}+1\right)$ on the right hand size, since $S_{[i]}$ is the sum of all monomials of degree $i$ (see e.g. [3] for a proof) and $\prod x_{i}^{\lambda_{i}}$ can be decomposed in exactly $\prod\left(\lambda_{i}+1\right)$ ways as a product of two monomials. 
Finally, by Pieri's rule (see [33, theorem 7.15.7 on page 339]), $S_{[i]} S_{[n-i]}$ is the sum of Schur polynomials of two-line and one-line diagrams, in which $S_{[a, b]}$ is a summand exactly when $b \leq i \leq a$. This shows (10) and hence clause (i) of the theorem.

Proof of (ii). For any $\pi \in T_{\lambda}$, let $\alpha_{k}^{(i)}(\pi)$ be the number of $k$-cycles in the $\operatorname{Sym}_{\lambda_{i}}{ }^{-}$ component of $\pi$ i.e. in the restriction of $\pi$ to $A_{\lambda}^{i}$. Clearly,

$$
\sum_{\pi \in T_{\lambda}} \alpha_{k}(\pi) 2^{\alpha(\pi)}=\sum_{\pi \in T_{\lambda}} \sum_{i: \lambda_{i} \geq k} \alpha_{k}^{(i)}(\pi) 2^{\alpha(\pi)}=\sum_{i: \lambda_{i} \geq k} \sum_{\pi \in T_{\lambda}} \alpha_{k}^{(i)}(\pi) 2^{\alpha(\pi)}
$$

To compute the inner sum, let us look at all the $k$-size subsets $A \subseteq A_{\lambda}^{i}$, and for each such $A$ let $T_{\lambda, A}$ be the set of permutations $\pi \in T_{\lambda}$ that preserve $A$ and $\left.\pi\right|_{A}$ is a $k$-cycle. Since there are $(k-1) ! k$-cycles in $\mathrm{Sym}_{k}$, we have

$$
\sum_{\pi \in T_{\lambda, A}} 2^{\alpha(\pi)}=2(k-1) !\left(\prod_{j \neq i}\left(\lambda_{j}+1\right) !\right)\left(\lambda_{i}-k+1\right) !
$$

Summing over all the possible $A$ 's, we get

$$
\sum_{\pi \in T_{\lambda}} \alpha_{k}^{(i)}(\pi) 2^{\alpha(\pi)}=\left(\begin{array}{c}
\lambda_{i} \\
k
\end{array}\right) 2(k-1) !\left(\prod_{j \neq i}\left(\lambda_{j}+1\right) !\right)\left(\lambda_{i}-k+1\right) !
$$

Summing over $i$ :

$$
\sum_{\pi \in T_{\lambda}} \alpha_{k}(\pi) 2^{\alpha(\pi)}=2 \sum_{i: \lambda_{i} \geq k}\left(\begin{array}{c}
\lambda_{i} \\
k
\end{array}\right)\left(\prod_{j \neq i}\left(\lambda_{j}+1\right) !\right)\left(\lambda_{i}-k+1\right) !(k-1) !
$$

Hence

$$
\begin{aligned}
\frac{1}{\left|T_{\lambda}\right|} \sum_{\pi \in T_{\lambda}} \alpha_{k}(\pi) 2^{\alpha(\pi)} & =2 \sum_{i: \lambda_{i} \geq k}\left(\begin{array}{c}
\lambda_{i} \\
k
\end{array}\right)\left(\prod_{j \neq i}\left(\lambda_{j}+1\right)\right) \frac{\left(\lambda_{i}-k+1\right) !(k-1) !}{\lambda_{i} !} \\
& =\frac{2}{k} \sum_{i: \lambda_{i} \geq k} \prod_{j} \begin{cases}\left(\lambda_{j}+1\right) & j \neq i \\
\left(\lambda_{j}+1-k\right) & j=i\end{cases}
\end{aligned}
$$

We conclude by (9), that

$$
\begin{aligned}
\operatorname{ch}\left(\alpha_{k} 2^{\alpha}\right) & =\frac{2}{k} \sum_{n \vdash \lambda} M_{\lambda} \sum_{i: \lambda_{i} \geq k} \prod_{j} \begin{cases}\left(\lambda_{j}+1\right) & j \neq i \\
\left(\lambda_{j}+1-k\right) & j=i\end{cases} \\
& =\frac{2}{k} u_{k} \sum_{n-k \vdash \lambda} M_{\lambda} \prod_{j}\left(\lambda_{j}+1\right)
\end{aligned}
$$

The last equality follows by comparing the coefficient of $\prod x_{i}^{\lambda_{i}}$ on both sides. The result now follows from (10).

The formula in theorem 4 expresses $\operatorname{ch}\left(\alpha_{k} 2^{\alpha}\right)$ as a product of $u_{k}$ and a linear combination of Schur polynomials. We will use a Murnaghan-Nakayama type formula to further simplify the expression and present it as a linear combination of Schur polynomials. Recall from the introduction the notions of a skew diagram and a border strip, and the notation $\operatorname{ht}(\mu)$. Let $E_{k}(\mu)$ be the set of Young diagrams $\lambda$ such that $\lambda \supseteq \mu$ and $\lambda \backslash \mu$ is a border strip of size $k$. We quote the following 
formula from Stanley's Enumerative Combinatorics (see [33, Theorem 7.17.1], but note that Stanley's ht differs from ours by 1 ):

$$
u_{k} S_{\mu}=\sum_{\lambda \in E_{k}(\mu)}(-1)^{\mathrm{ht}(\lambda \backslash \mu)+1} S_{\lambda}
$$

We therefore have

$$
\operatorname{ch}\left(\alpha_{k} 2^{\alpha}\right)=\frac{2}{k} \sum_{n-k \vdash \mu=[a, b]}(a-b+1) \sum_{\lambda \in E_{k}(\mu)}(-1)^{\mathrm{ht}(\lambda \backslash \mu)+1} S_{\lambda}
$$

concluding the proof of theorem 3.

\section{The EXPECTATION OF $\alpha_{k} 2^{\alpha}$ AT time $t$}

Let us see how the decomposition in theorem 3 helps us calculate $\mathbb{E}\left(\alpha_{k}(\pi(t))\right.$. $\left.2^{\alpha(\pi(t))}\right)$. We have the following general lemma:

Lemma 5. Let $\chi_{\lambda}$ be an irreducible character of $S_{n}, R_{\lambda}$ the corresponding representation, $B=\left(b_{i j}\right)$ a symmetric $n \times n$ matrix with positive entries outside the diagonal, $\left(\pi^{B}(t) \in S_{n}\right)_{t \geq 0}$ the continuous time interchange process with rates $\left(b_{i j}\right)$, and $\rho_{1}, \ldots, \rho_{d}$ the eigenvalues of $\sum_{i<j} b_{i j}\left(i d-R_{\lambda}((i j))\right)$. Then

$$
\mathbb{E}\left(\chi_{\lambda}\left(\pi^{B}(t)\right)\right)=\sum_{i} e^{-t \rho_{i}}
$$

Proof. The arguments in the proof of [3, lemma 5] hold in this generality. For the sake of brevity, we will not repeat them.

Let us get back to the analysis of the mean field case, $(\pi(t))_{t \geq 0}$, for which the rates are $a_{i j}=1$. Let $n \vdash \lambda$. As mentioned in $\S 1.3$, the eigenvalues of $\widehat{\Delta}(\lambda)$ are all equal to

$$
\rho(\lambda)=\left(\begin{array}{l}
n \\
2
\end{array}\right)-\left(\begin{array}{l}
n \\
2
\end{array}\right) \frac{\chi_{\lambda}((12))}{d_{\lambda}} .
$$

Definition 6. For any box $(i, j)$ of the diagram $\lambda$, the content of the box is defined by

$$
c((i, j))=i-j
$$

Here and below, $i$ is the row index and $j$ is the position inside the row, both starting from 1.

Lemma 7. Let $n \vdash \lambda$. We have

$$
\left(\begin{array}{l}
n \\
2
\end{array}\right) \frac{\chi_{\lambda}((12))}{d_{\lambda}}=-\sum_{(i, j) \in \lambda} c((i, j))
$$

Proof. By the formula in [16, lemma 7],

$$
\begin{aligned}
\left(\begin{array}{l}
n \\
2
\end{array}\right) \frac{\chi_{\lambda}((12))}{d_{\lambda}} & =\frac{1}{2} \sum_{i=1}^{r}\left(\left(\lambda_{i}-i\right)\left(\lambda_{i}-i+1\right)-i(i-1)\right)= \\
& =\sum_{i=1}^{r} \frac{\lambda_{i}\left(\lambda_{i}+1\right)}{2}-\sum_{i=1}^{r} i \lambda_{i}= \\
& =\sum_{(i, j) \in \lambda} j-\sum_{(i, j) \in \lambda} i=-\sum_{(i, j) \in \lambda} c((i, j)) .
\end{aligned}
$$


We therefore have

$$
\rho(\lambda)=\left(\begin{array}{l}
n \\
2
\end{array}\right)+\sum_{(i, j) \in \lambda} c((i, j))
$$

and (by theorem 3 and lemma 5),

$$
\mathbb{E}\left(\alpha_{k}(\pi(t)) 2^{\alpha(\pi(t))}\right)=\frac{2}{k} \sum_{n-k \vdash \mu=[a, b]}(a-b+1) \sum_{\lambda \in E_{k}(\mu)}(-1)^{h t(\lambda \backslash \mu)+1} d_{\lambda} e^{-t \rho(\lambda)}
$$

\section{The Caterpillar}

For a Young diagram $\mu=\left[\mu_{1}, \ldots, \mu_{r}\right]$, we have denoted by $E_{k}(\mu)$ the set of Young diagrams $\lambda$ which contain $\mu$, and such that $\lambda \backslash \mu$ is a border strip of size $k$. Here is for example the set $E_{3}([5,3])$ :
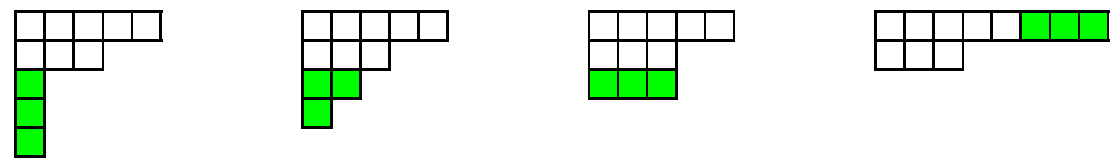

Figure 1. $E_{3}([5,2])$

Pictorially, we like to think of $E_{k}(\mu)$ as the result of the following process: We start with the diagram $\left[\mu_{1}, \mu_{2}, \ldots, \mu_{r}, 1^{k}\right]$. We view this diagram as the diagram $\mu$ with a caterpillar with $k$ square segments, lying below it. The top square $(r+1,1)$ is the head of the caterpillar. At each stage the caterpillar moves forward one step. Each segment moves to the place occupied by the next one, whereas the head moves either up or right, so that the caterpillar clings to $\mu$. The caterpillar stops when it lies entirely in the first row (i.e. when the diagram is $\left[\mu_{1}+k, \mu_{2}, \ldots, \mu_{r}\right]$. Some of the diagrams we obtain are not Young diagrams, but the set of Young diagrams obtained in this process is precisely $E_{k}(\mu)$.
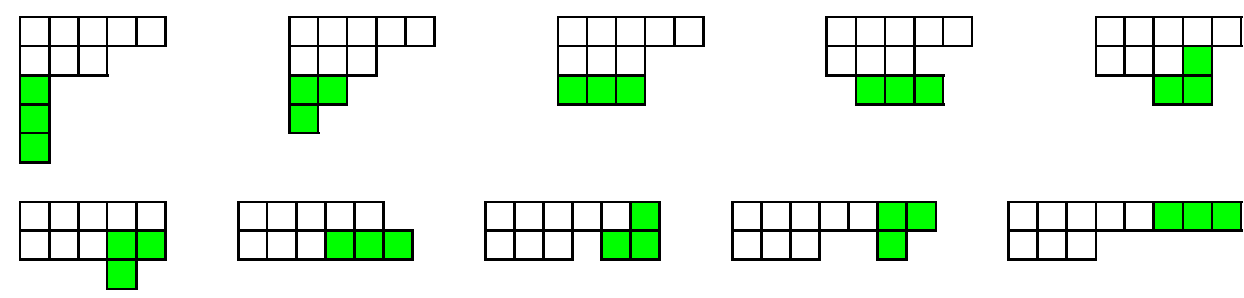

Figure 2. Caterpillar moves

We may narrow down the list of caterpillar positions by requiring that the tail of the caterpillar is aligned to the left. For any $1 \leq i \leq r+k$, we look at the caterpillar configuration for which the tail lies at $\left(i, \mu_{i}+1\right)$ (where $\mu_{j}=0$ for $j>r)$. We may obtain this configuration as follows: We start with the diagram $\left[\mu_{1}, \ldots, \mu_{i}+k, \ldots, \mu_{r}\right]$ (when $i>r$ this means $\left[\mu_{1}, \ldots, \mu_{r}, 0^{i-r-1}, k\right]$ ). This is not necessarily a Young diagram, as the sequence $\mu_{1}, \ldots, \mu_{i}+k, \ldots, \mu_{r}$ may not be 
nonincreasing. We perform a sequence of moves to make it a Young diagram. In each move, we replace a pair of row lengths $\mu_{j}, \mu_{j+1}$ (where $\mu_{j+1} \geq \mu_{j}+2$ ) with the pair $\mu_{j+1}-1, \mu_{j}+1$ (pictorially, wrapping the $j+1^{\text {th }}$ row around the corner above it). We stop when no move is applicable.

Here is an illustration for $\mu=[3,2], k=5$ and $i=4$ :
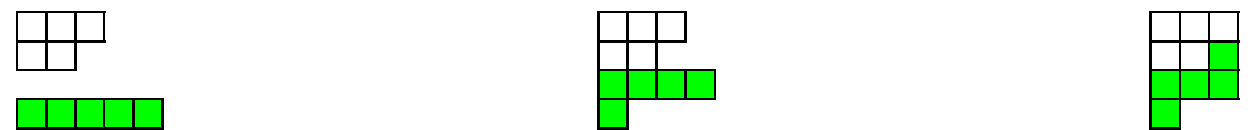

FiguRE 3. The wrapping process

We now formalize the above description.

Definition 8. We call a diagram of the form $\mu=\left[\mu_{1}, \ldots, \mu_{r}\right]$ a pre-diagram if the numbers $\mu_{1}, \ldots, \mu_{r}$ are nonnegative integers, $\mu_{r} \neq 0$, and we have

$$
\left|\left\{i \in\{1, \ldots, r-1\}: \mu_{i+1}>\mu_{i}\right\}\right| \leq 1 \text {. }
$$

Note that any Young diagram is also a pre-diagram.

Let $R_{j}$ be the move which replaces a pre-diagram $\mu=\left[\mu_{1}, \ldots, \mu_{r}\right]$ with $\left[\mu_{1}, \ldots\right.$, $\left.\mu_{j-1}, \mu_{j+1}-1, \mu_{j}+1, \mu_{j+2}, \ldots, \mu_{r}\right]$. This move is only applicable when $j \leq r$ and $\mu_{j+1} \geq \mu_{j}+2$. Note that the resulting sequence is still a pre-diagram.

Lemma 9. Let $\mu=\left[\mu_{1}, . ., \mu_{r}\right]$ be a pre-diagram. Consider the following process, called the wrapping process, which generates a sequence of pre-diagrams $\mu(0)$, $\mu(1), \ldots$ : We start with $\mu(0)=\mu$. Given $\mu(j), \mu(j+1)$ is obtained from it by one of the moves $R_{l}$. The process terminates when no move can be applied. Then:

(i) At each stage, there is either one or no move that can be applied. Thus, the sequence $\mu(0), \mu(1), \ldots$ is uniquely determined.

(ii) The sequence $\mu(0), \mu(1), \ldots$ is finite.

Proof. The first assertion is obvious, since for a pre-diagram, there is at most one index $j$ for which $\mu_{j+1}>\mu_{j}$. The second assertion is obvious as well, since the index $j$ for $\mu_{j+1}>\mu_{j}$ (if it exists) drops by 1 after each application of a move.

Let us denote by $Y(\mu)$ the final pre-diagram achieved by the wrapping process starting from $\mu$.

Further, for a Young diagram $\mu=\left[\mu_{1}, \ldots, \mu_{r}\right]$, and positive integers $i$ and $k$, let $F_{k, i}(\mu)$ be the diagram

$$
F_{k, i}(\mu)= \begin{cases}{\left[\mu_{1}, \ldots, \mu_{i}+k, \ldots, \mu_{r}\right]} & i \leq r \\ {\left[\mu_{1}, \ldots, \mu_{r}, 0^{i-r-1}, k\right]} & i>r\end{cases}
$$

Note that $F_{k, i}(\mu)$ is a pre-diagram.

Lemma 10. Let $n-k \vdash \mu=\left[\mu_{1}, \ldots, \mu_{r}\right], k>0,1 \leq i \leq r+k$, and let $\delta=$ $Y\left(F_{k, i}(\mu)\right)$. Then:

(i) $\delta$ is a Young diagram if and only if there exists $\lambda \in E_{\mu, k}$ such that the lowest row of $\lambda \backslash \mu$ is positioned at $i$. In that case, such $\lambda$ is unique: $\lambda=\delta$. 
(ii) Let $m$ be the total number of moves in the wrapping process on $F_{k, i}(\mu)$. Then $m=\operatorname{ht}(\delta \backslash \mu)-1$.

Proof. Let $\lambda=F_{k, i}(\mu)$ and let $\lambda=\lambda(0), \lambda(1), \ldots, \lambda(m)=\delta$ be the stages of the wrapping process. It can be shown by induction that for all $j$,

$$
\lambda(j)=\left[\mu_{1}, \ldots, \mu_{i-j-1},\left(\mu_{i}+k-j\right), \mu_{i-j}+1, \ldots, \mu_{i-1}+1, \mu_{i+1}, \ldots, \mu_{r}\right]
$$

and $u_{i}+k-j \geq u_{i-j}+1$. The only move that can be applied on $\lambda(j)$ (if any) is $R_{i-j-1}$. The process terminates when $R_{i-j-1}$ can not be applied to $\lambda(j)$, i.e., when

$$
\mu_{i}+k-j \leq \mu_{i-j-1}+1
$$

which is equivalent to

$$
\mu_{i-j-1}+j \geq \mu_{i}+k-1
$$

hence,

$$
\mu_{i-m-1}+m \geq \mu_{i}+k-1
$$

Let $B_{\mu, i, k}$ be the set of pre-diagrams $\beta$ such that $\beta \backslash \mu$ is a border strip of size $k$, whose lowest row is at $i$. We claim that $B_{\mu, i, k}=\{\lambda(0), \ldots, \lambda(m)\}$. Indeed, any $\beta \in B_{\mu, i, k}$ must be of the form $\left[\mu_{1}, \ldots, \mu_{i-j-1}, \beta_{i-j}, \ldots, \beta_{i}, \mu_{i+1}, \ldots, \mu_{r}\right]$ for some $j \geq 0$ and some numbers $\beta_{i-j} \geq \beta_{i-j+1} \ldots \geq \beta_{i}$ satisfying $\beta_{l}>\mu_{l}$ for all $l$, and

$$
\sum_{l=i-j}^{i}\left(\beta_{l}-\mu_{l}\right)=k
$$

By the border strip conditions, we must have for all $i-j<l \leq i, \beta_{l} \geq \mu_{l-1}+1$ (otherwise $\beta \backslash \mu$ would not be connected), and $\beta_{l}<\mu_{l-1}+2$ (otherwise we would have $\beta_{l-1} \geq \beta_{l} \geq \mu_{l-1}+2$ and then $\beta \backslash \mu$ would contain a $2 \times 2$ square, namely $\{l-1, l\} \times\left\{\mu_{l-1}+1, \mu_{l-1}+2\right\}$, contrary to the border strip conditions). Hence $\beta_{l}=\mu_{l-1}+1$ for all such $l$, and therefore $\beta_{i-j}=\mu_{i}+k-j$ by (16). We must have $j \leq m$, as otherwise, we would have by $(15), \mu_{i-j}+(j-1) \geq \mu_{i-m-1}+m \geq \mu_{i}+k-1$, and consequently, $\beta_{i-j}=\mu_{i}+k-j \leq \mu_{i-j}$, a contradiction. We have therefore verified that $B_{\mu, i, k} \subseteq\{\lambda(0), . ., \lambda(m)\}$. In the other direction, it is straightforward to check that $\lambda(j) \in B_{\mu, i, k}$ for all $0 \leq j \leq m$. This shows our claim.

Clearly, $\lambda(0), \ldots, \lambda(m-1)$ are not Young diagrams, as none of the moves is applicable on a Young diagram. We conclude that $B_{\mu, i, k} \cap E_{\mu, k} \subseteq\{\lambda(m)\}=\{\delta\}$, with equality if and only if $\delta$ is a Young diagram. This proves (i). Finally, by equation $(14), \operatorname{ht}(\lambda(j) \backslash \mu)=j+1$ for all $j$. Setting $j=m$, we get $(i i)$.

Corollary 11. Let $\mathbb{Y}$ be the set of all Young diagrams. Then

$$
E_{\mu, k}=\left\{Y\left(F_{k, i}(\mu)\right) \mid 1 \leq i \leq \mathrm{ht}(\mu)+k\right\} \cap \mathbb{Y}
$$

We will use this parametrization of $E_{\mu, k}$ in the evaluation of formula (13). As a first step, let us evaluate the laplacian eigenvalue $\rho\left(Y\left(F_{k, i}(\mu)\right)\right)$ :

Lemma 12. Assume that $Y\left(F_{k, i}(\mu)\right)$ is a Young diagram, where $n-k \vdash \mu$. We have

$$
\rho\left(Y\left(F_{k, i}(\mu)\right)\right)=\left(\begin{array}{l}
n \\
2
\end{array}\right)+\sum_{(r, s) \in \mu} c((r, s))+k\left(i-\mu_{i}-\frac{k+1}{2}\right)
$$


Proof. We observe that the sum $\sum_{(r, s) \in \lambda} c(r, s)$ is invariant under the moves $R_{l}$ (as each such move is equivalent to moving some boxes in the up-left direction, and $c((r, s))=c((r-1, s-1))$ for all $r, s)$. Since $Y\left(F_{k, i}(\mu)\right)$ is obtained from $F_{k, i}(\mu)$ by such moves, we can plug $F_{k, i}(\mu)=\left[\mu_{1}, \ldots, \mu_{i}+k, \ldots, \mu_{r}\right]$ in formula (12) and obtain $\rho\left(Y\left(F_{k, i}(\mu)\right)\right)$ :

$$
\rho\left(Y\left(F_{k, i}(\mu)\right)\right)=\left(\begin{array}{l}
n \\
2
\end{array}\right)+\sum_{(r, s) \in \mu} c((r, s))+\sum_{s=\mu_{i}+1}^{\mu_{i}+k} c((i, s))
$$

The result follows by evaluating the arithmetic sum in the last term.

\section{THE HOOK PARAMETRIZATION}

Definition 13. For a pre-diagram $\lambda=\left[\lambda_{1}, \lambda_{2}, \ldots, \lambda_{r}\right]$ (where $\lambda_{i} \geq 0, \lambda_{r}>0$ ) we call

$$
\lambda^{(i)}=\lambda_{i}+r-i \quad i=1,2, \ldots, r
$$

the hook numbers of $\lambda$.

If $\lambda$ is a Young diagram, then $\lambda^{(i)}$ is the length of the hook whose corner is the box $(i, 1)$. Also, for a Young diagram, since $\lambda_{1} \geq \lambda_{2} \geq \ldots \lambda_{r} \geq 0$, the numbers $\lambda^{(i)}$ satisfy $\lambda^{(1)}>\lambda^{(2)}>\cdots>\lambda^{(r)}>0$, and in particular, $\lambda^{(i)} \neq \lambda^{(j)}$ for $i \neq j$. As we shall see, representing diagrams by their hook numbers leads to a considerable simplification of formula (13).

Lemma 14. Let $\lambda=\left[\lambda_{1}, \ldots, \lambda_{r}\right]$ be a pre-diagram, and let $\delta=Y(\lambda)$.

(i) $\lambda$ and $\delta$ have the same hook lengths (possibly in a different order).

(ii) $\delta$ is a Young diagram if and only if $\lambda^{(i)} \neq \lambda^{(j)}$ for all $i \neq j$.

Proof.

(i) Since $\delta$ is obtained from $\lambda$ by a sequence of the moves $\left\{R_{j}\right\}$, it is enough to show that any such move preserves the multiset of hook lengths. Indeed, the move $R_{j}$ replaces $\lambda_{j}, \lambda_{j+1}$ by $\lambda_{j+1}-1, \lambda_{j}+1$, while leaving the other row lengths intact. Thus the pair $\lambda^{(j)}, \lambda^{(j+1)}$ is replaced by $\lambda^{(j+1)}, \lambda^{(j)}$, and the multiset $\left\{\lambda^{(1)}, \ldots, \lambda^{(r)}\right\}$ is preserved.

(ii) If $\delta$ is a Young diagram, then we have $\delta^{(i)} \neq \delta^{(j)}$ for $i \neq j$, and by (i), $\lambda^{(i)} \neq \lambda^{(j)}$ for all $i \neq j$. In the other direction, if $\lambda^{(i)} \neq \lambda^{(j)}$ for all $i \neq j$ then again by (i), we have $\delta^{(i)} \neq \delta^{(j)}$ for $i \neq j$. Moreover, none of the moves $\left\{R_{j}\right\}$ is applicable to $\delta$, so $\delta_{j+1} \leq \delta_{j}+1$ for all $1 \leq j \leq r-1$. Since $\delta^{(j+1)} \neq \delta^{(j)}$ for any such $j$, we have $\delta_{j+1} \neq \delta_{j}+1$. Hence $\delta_{1} \geq \delta_{2} \geq \ldots \geq$ $\delta_{r}$, and $\delta$ is a Young diagram.

For any Young diagram $\lambda=\left[\lambda_{1}, \ldots, \lambda_{m}\right]$, there is a well-known formula for the dimension $d_{\lambda}$ in terms of the hook numbers $\lambda^{(i)}$ :

Lemma 15. (The Young-Frobenius formula).

$$
d_{\lambda}=\frac{n !}{\prod_{t=1}^{m} \lambda^{(t) !}} \prod_{1 \leq t<s \leq m}\left(\lambda^{(t)}-\lambda^{(s)}\right)
$$

This formula was discovered by Frobenius [18] and independently by Young [38]. It is not difficult to see its equivalence to the more familiar hook formula.

We can now evaluate the numbers $(-1)^{h t(\lambda \backslash \mu)+1} d_{\lambda}$ which appear in formula (13): 
Theorem 16. Let $\mu=\left[\mu_{1}, \ldots, \mu_{r}\right], \lambda=F_{k, i}(\mu)$ and $\delta=Y(\lambda)$. Let $m=h t(\delta)=$ $h t(\lambda)=\max \{i, r\}$. We have

$$
\frac{n !}{\prod_{t=1}^{m} \lambda^{(t) !}} \prod_{1 \leq t<s \leq m}\left(\lambda^{(t)}-\lambda^{(s)}\right)= \begin{cases}(-1)^{h t(\delta \backslash \mu)+1} d_{\delta} & \delta \text { is a Young diagram } \\ 0 & \text { otherwise }\end{cases}
$$

Proof. By lemma 14, $\delta$ is a Young diagram if and only if all the numbers $\lambda^{(1)}, \ldots$, $\lambda^{(m)}$ are different from one another. Let us assume that $\delta$ is indeed a Young diagram. Since $\left\{\delta^{(1)}, \ldots, \delta^{(m)}\right\}=\left\{\lambda^{(1)}, \ldots, \lambda^{(m)}\right\}$, by the Young-Frobenius formula we have

$$
d_{\lambda}= \pm \frac{n !}{\prod_{t=1}^{m} \lambda^{(t) !}} \prod_{1 \leq t<s \leq m}\left(\lambda^{(t)}-\lambda^{(s)}\right)
$$

The sign in this formula is the sign of the permutation which takes $\left\{\delta^{(1)}, \ldots, \delta^{(m)}\right\}$ to $\left\{\lambda^{(1)}, \ldots, \lambda^{(m)}\right\}$. We have seen in the proof of lemma 14 that each move $R_{j}$ applied to a pre-diagram induces a switch of two consecutive values in the corresponding hook numbers. By lemma 10, it takes $\mathrm{ht}(\lambda \backslash \mu)-1=\mathrm{ht}(\delta \backslash \mu)-1$ moves to get from $\lambda$ to $\delta$. This proves the formula.

6. The expectation of $\alpha_{k} 2^{\alpha}$ at time $t$, Continued

We are now ready to complete the calculation of $\mathbb{E}\left(\alpha_{k}(\pi(t)) 2^{\alpha(\pi(t))}\right)$.

Theorem 17. Let $y=e^{-t k}$. Then

$$
\mathbb{E}\left(\alpha_{k}(\pi(t)) 2^{\alpha(\pi(t))}\right)=\frac{2}{k} \sum_{n-k \vdash[a, b]}(a+1-b) \phi([a, b], k, t)
$$

where $\phi$ is given, for $b>0$, by

$$
\begin{aligned}
\phi([a, b], k, t)=\left(\begin{array}{c}
n \\
k-1, a+1, b
\end{array}\right) \cdot e^{-t(a b+b)}\left[\frac{(a+1-b) y^{a+b+2}(1-y)^{k-1}}{k}\right. \\
+y^{b}(a+1-b+k) \int_{y}^{1} x^{a+1}(1-x)^{k-1} d x \\
\left.+y^{a+1}(a+1-b-k) \int_{y}^{1} x^{b}(1-x)^{k-1} d x\right]
\end{aligned}
$$

and for $b=0$ by

$$
\phi([n-k], k, t)=\left(\begin{array}{l}
n \\
k
\end{array}\right)\left[y^{n-k+1}(1-y)^{k-1}+k \int_{y}^{1} x^{n-k}(1-x)^{k-1} d x\right]
$$

Proof. Let us repeat equation (13):

$$
\mathbb{E}\left(\alpha_{k}(\pi(t)) 2^{\alpha(\pi(t))}\right)=\frac{2}{k} \sum_{n-k \vdash \mu=[a, b]}(a-b+1) \sum_{\lambda \in E_{k}(\mu)}(-1)^{\mathrm{ht}(\lambda \backslash \mu)+1} d_{\lambda} e^{-t \rho(\lambda)}
$$

where $E_{k}(\mu)$ is the set of $\lambda \dashv n$ such that $\mu \subset \lambda$ and $\lambda \backslash \mu$ is a border strip of size $k$.

We now fix some $n-k \vdash \mu=[a, b]$, and denote the inner sum in the above equation by

$$
\tau(\mu, k, t)=\sum_{\lambda \in E_{k}(\mu)}(-1)^{h t(\lambda \backslash \mu)+1} d_{\lambda} e^{-t \rho(\lambda)}
$$


Our goal is to prove that $\tau(\mu, k, t)=\phi(\mu, k, t)$. We treat the case $b>0$ first.

By corollary 11 and theorem 16 , we may write $\tau(\mu, k, t)$ as

$$
\tau(\mu, k, t)=\sum_{i=1}^{k+2} D(\mu, k, i) \exp \left(-t \rho\left(Y\left(F_{k, i}(\mu)\right)\right)\right)
$$

where

$$
\begin{aligned}
D(\mu, k, i) & = \begin{cases}(-1)^{h t\left(Y\left(F_{k, i}(\mu) \backslash \mu\right)\right)+1} d_{Y\left(F_{k, i}(\mu)\right)} & Y\left(F_{k, i}(\mu)\right) \text { is a Young diagram } \\
0 & \text { otherwise }\end{cases} \\
& =\frac{n !}{\prod_{t=1}^{m} \gamma^{(t) !}} \prod_{1 \leq t<s \leq m}\left(\gamma^{(t)}-\gamma^{(s)}\right)
\end{aligned}
$$

and where $\gamma=\left[\gamma_{1}, \ldots, \gamma_{m}\right]=F_{k, i}(\mu)$. Note that $\rho\left(Y\left(F_{k, i}(\mu)\right)\right)$ was not defined when $Y\left(F_{k, i}(\mu)\right)$ is not a Young diagram - for some values of $i$ and $k$ it may just be a prediagram - but in such cases, it is multiplied by zero in (18), so we may define it arbitrarily. It turns out that the cases $i=1$ and 2 are best handled separately, so write

$$
\tau(\mu, k, t)=\mathrm{I}+\mathbb{I}+\mathbb{I I I}
$$

where $\mathbf{I}=D(\mu, k, 1) \exp \left(-t \rho\left(Y\left(F_{k, 1}(\mu)\right)\right)\right), \mathbb{I}=D(\mu, k, 2) \exp \left(-t \rho\left(Y\left(F_{k, 2}(\mu)\right)\right)\right)$ and $\mathrm{III}$ is the sum over the remaining terms.

Let us consider a few cases and write $\gamma$ and its vector of hook lengths, $\left(\gamma^{(1)}, \ldots\right.$, $\left.\gamma^{(m)}\right)$ in each case:

$$
\gamma= \begin{cases}{[a+k, b]} & i=1 \\ {[a, b+k]} & i=2 \\ {\left[a, b, 0^{i-3}, k\right]} & i>2\end{cases}
$$

and consequently

$$
\left(\gamma^{(1)}, \ldots, \gamma^{(m)}\right)= \begin{cases}(a+k+1, b) & i=1 \\ (a+1, b+k) & i=2 \\ (a+i-1, b+i-2, i-3, i-4, \ldots, 1, k) & i>2\end{cases}
$$

We conclude:

$$
D(\mu, k, i)= \begin{cases}\frac{n !(a+k+1-b)}{(a+k+1) ! b !} & i=1 \\ \frac{n !(a+1-b-k)}{(a+1) !(b+k) !} & i=2\end{cases}
$$


while for $i>2$ the main term is

$$
\begin{aligned}
& \prod_{1 \leq t<s \leq m}\left(\gamma^{(t)}-\gamma^{(s)}\right)=(a+1-b)\left(\prod_{j=a+2}^{a+i-2} j\right)(a+i-1-k)\left(\prod_{j=b+1}^{b+i-3} j\right) \\
& \cdot(b+i-2-k)\left(\prod_{j=1}^{i-4} j !\right)\left(\prod_{j=1-k}^{i-3-k} j\right) \\
&=(a+1-b)(a+i-1-k)(b+i-2-k) \\
& \frac{(a+i-2) !}{(a+1) !} \frac{(b+i-3) !}{b !}\left(\prod_{j=1}^{i-4} j !\right)(-1)^{i-3} \frac{(k-1) !}{(k+2-i) !}
\end{aligned}
$$

and the term in the denominator is

$$
\prod_{t=1}^{m} \gamma^{(t)} !=(a+i-1) !(b+i-2) !\left(\prod_{j=1}^{i-3} j !\right) k !
$$

Hence, after some simplification,

$$
\begin{aligned}
& D(\mu, k, i)= \\
& \frac{a+1-b}{k}\left(\begin{array}{c}
n \\
k-1, a+1, b
\end{array}\right)(-1)^{i+1}\left(\begin{array}{c}
k-1 \\
i-3
\end{array}\right) \frac{(a+i-1-k)(b+i-2-k)}{(a+i-1)(b+i-2)} .
\end{aligned}
$$

By a partial fraction decomposition,

$$
\begin{aligned}
& \frac{(a+i-1-k)(b+i-2-k)}{(a+i-1)(b+i-2)}= \\
& =\left(1-\frac{k}{a+i-1}\right)\left(1-\frac{k}{b+i-2}\right) \\
& =1-\frac{k}{a+i-1}-\frac{k}{b+i-2}+\frac{k^{2}}{(a+i-1)(b+i-2)} \\
& =1-\frac{k}{a+i-1}-\frac{k}{b+i-2}-\frac{k^{2}}{a+1-b}\left(\frac{1}{a+i-1}-\frac{1}{b+i-2}\right) \\
& =\frac{1}{a+1-b}\left((a+1-b)-\frac{k(a+1-b+k)}{a+i-1}-\frac{k(a+1-b-k)}{b+i-2}\right)
\end{aligned}
$$

so

$$
\begin{aligned}
D(\mu, k, i) & =\frac{1}{k}\left(\begin{array}{c}
n \\
k-1, a+1, b
\end{array}\right)(-1)^{i+1}\left(\begin{array}{c}
k-1 \\
i-3
\end{array}\right) \\
& \cdot\left((a+1-b)-\frac{k(a+1-b+k)}{a+i-1}-\frac{k(a+1-b-k)}{b+i-2}\right)
\end{aligned}
$$

Next, let us calculate the eigenvalue $\rho\left(Y\left(F_{k, i}(\mu)\right)\right)$. We have

$$
\sum_{(i, j) \in \mu=[a, b]} c((i, j))=-\frac{a(a-1)}{2}-\frac{(b-1)(b-2)}{2}+1
$$


and by lemma 12 ,

$$
\begin{aligned}
\rho\left(Y\left(F_{k, i}(\mu)\right)\right)= & \left(\begin{array}{l}
n \\
2
\end{array}\right)+\sum_{(i, j) \in \mu=[a, b]} c((i, j))+k\left(i-\mu_{i}-\frac{k+1}{2}\right) \\
= & \frac{n(n-1)}{2}-\frac{a(a-1)}{2}-\frac{(b-1)(b-2)}{2}-\frac{k(k+1)}{2}+1+k i-k \mu_{i} \\
= & k i-k \mu_{i}+\frac{1}{2}(2+(a+b+k)(a+b+k-1) \\
& \quad-a(a-1)-(b-1)(b-2)-k(k+1)) \\
= & k i-k \mu_{i}+a b+a k+b k+b-k .
\end{aligned}
$$

For $i>2$, we have $\mu_{i}=0$, and we conclude that

$$
\exp \left(-t \rho\left(Y\left(F_{k, i}(\mu)\right)\right)\right)=e^{-t k i} e^{-t(a b+a k+b k+b-k)}
$$

Let us put everything together:

$$
\begin{aligned}
\text { III } & =\sum_{i=3}^{k+2} D(\mu, k, i) \exp \left(-t \rho\left(Y\left(F_{k, i}(\mu)\right)\right)\right) \\
& =e^{-t(a b+a k+b k+b-k)} \frac{1}{k}\left(\begin{array}{c}
n \\
k-1, a+1, b
\end{array}\right) . \\
& \cdot \sum_{i=3}^{k+2}(-1)^{i+1}\left(\begin{array}{c}
k-1 \\
i-3
\end{array}\right) e^{-t k i}\left((a+1-b)-\frac{k(a+1-b+k)}{a+i-1}-\frac{k(a+1-b-k)}{b+i-2}\right)
\end{aligned}
$$

Let us move $e^{-3 t k}$ outside, and replace $i-3$ with $i$ :

$$
\begin{aligned}
& \text { III }=e^{-t(a b+a k+b k+b-k)} \frac{1}{k}\left(\begin{array}{c}
n \\
k-1, a+1, b
\end{array}\right) \cdot e^{-3 t k} \\
& \cdot \sum_{i=0}^{k-1}(-1)^{i}\left(\begin{array}{c}
k-1 \\
i
\end{array}\right) e^{-t k i}\left((a-b+1)-\frac{k(a+1-b+k)}{a+i+2}-\frac{k(a+1-b-k)}{b+i+1}\right)
\end{aligned}
$$

Let us evaluate the inner sum. For the first summand we use the binomial formula:

$$
\sum_{i=0}^{k-1}(-1)^{i}\left(\begin{array}{c}
k-1 \\
i
\end{array}\right) e^{-t k i}=\left(1-e^{-t k}\right)^{k-1}
$$

For the other two terms, we use integration and the binomial formula:

$$
\begin{aligned}
\sum_{i=0}^{k-1}(-1)^{i}\left(\begin{array}{c}
k-1 \\
i
\end{array}\right) \frac{e^{-t k i}}{a+i+2} & =e^{t k(a+2)} \sum_{i=0}^{k-1}(-1)^{i}\left(\begin{array}{c}
k-1 \\
i
\end{array}\right) \frac{e^{-t k(a+i+2)}}{a+i+2} \\
& =e^{t k(a+2)} \sum_{i=0}^{k-1}(-1)^{i}\left(\begin{array}{c}
k-1 \\
i
\end{array}\right) \int_{0}^{e^{-t k}} x^{a+i+1} d x \\
& =e^{t k(a+2)} \int_{0}^{e^{-t k}} x^{a+1} \sum_{i=0}^{k-1}\left(\begin{array}{c}
k-1 \\
i
\end{array}\right)(-x)^{i} d x \\
& =e^{t k(a+2)} \int_{0}^{e^{-t k}} x^{a+1}(1-x)^{k-1} d x
\end{aligned}
$$


and similarly,

$$
\sum_{i=0}^{k-1}(-1)^{i}\left(\begin{array}{c}
k-1 \\
i
\end{array}\right) \frac{e^{-t k i}}{b+i+1}=e^{t k(b+1)} \int_{0}^{e^{-t k}} x^{b}(1-x)^{k-1} d x
$$

Collecting the 3 terms, we obtain

$$
\begin{aligned}
& \text { III }=e^{-t(a b+a k+b k+b+2 k)} \frac{1}{k}\left(\begin{array}{c}
n \\
k-1, a+1, b
\end{array}\right) \cdot\left[(a+1-b)\left(1-e^{-t k}\right)^{k-1}\right. \\
&-k(a+1-b+k) e^{t k(a+2)} \int_{0}^{e^{-t k}} x^{a+1}(1-x)^{k-1} d x \\
&\left.-k(a+1-b-k) e^{t k(b+1)} \int_{0}^{e^{-t k}} x^{b}(1-x)^{k-1} d x\right]
\end{aligned}
$$

and with the notation $y=e^{-t k}$ :

$$
\begin{aligned}
\text { III }= & e^{-t(a b+b)}\left(\begin{array}{c}
n \\
k-1, a+1, b
\end{array}\right) \cdot\left[\frac{a+1-b}{k}(1-y)^{k-1} y^{a+b+2}\right. \\
& -(a+1-b+k) y^{b} \int_{0}^{y} x^{a+1}(1-x)^{k-1} d x \\
& \left.-(a+1-b-k) y^{a+1} \int_{0}^{y} x^{b}(1-x)^{k-1} d x\right] .
\end{aligned}
$$

Let us now add the terms I and $\mathbb{I}$. By (19) and (21),

$$
\begin{aligned}
\mathrm{I} & =D(\mu, k, 1) \exp \left(-t \rho\left(Y\left(F_{k, 1}(\mu)\right)\right)\right) \\
& =\frac{n !(a+k+1-b)}{(a+k+1) ! b !} e^{-t(a b+b k+b)} \\
& =\left(\begin{array}{c}
n \\
k-1, a+1, b
\end{array}\right) \frac{(a+1) !(k-1) !(a+k+1-b)}{(a+k+1) !} e^{-t(a b+b)} y^{b}
\end{aligned}
$$

and

$$
\begin{aligned}
\mathbb{I} & =D(\mu, k, 2) \exp \left(-t \rho\left(Y\left(F_{k, 2}(\mu)\right)\right)\right) \\
& =\frac{n !(a+1-b-k)}{(a+1) !(b+k) !} e^{-t(k+a b+a k+b)} \\
& =\left(\begin{array}{c}
n \\
k-1, a+1, b
\end{array}\right) \frac{b !(k-1) !(a+1-b-k)}{(b+k) !} e^{-t(a b+b)} y^{a+1}
\end{aligned}
$$


Collecting terms, we get the following formula for the inner sum in equation (13) (see also equation (18)):

$$
\begin{aligned}
& \tau(\mu, k, t)=\left(\begin{array}{c}
n \\
k-1, a+1, b
\end{array}\right) e^{-t(a b+b)} \cdot\left[\frac{(a+1-b)}{k} y^{a+b+2}(1-y)^{k-1}\right. \\
&-(a+1-b+k) y^{b} \int_{0}^{y} x^{a+1}(1-x)^{k-1} d x \\
&-(a+1-b-k) y^{a+1} \int_{0}^{y} x^{b}(1-x)^{k-1} d x \\
&+\frac{(a+1) !(k-1) !(a+k+1-b)}{(a+k+1) !} y^{b} \\
&\left.+\frac{b !(k-1) !(a+1-b-k)}{(b+k) !} y^{a+1}\right]
\end{aligned}
$$

We have the following Beta function identity:

$$
\int_{0}^{1} x^{a+1}(1-x)^{k-1} d x=B(a+2, k)=\frac{(k-1) !(a+1) !}{(a+k+1) !}
$$

Hence we may group the second and fourth summands in (22):

$$
\begin{aligned}
-(a+1- & b+k) y^{b} \int_{0}^{y} x^{a+1}(1-x)^{k-1} d x+\frac{(a+1) !(k-1) !(a+k+1-b)}{(a+k+1) !} y^{b} \\
& =(a+1-b+k) y^{b}\left(\int_{0}^{1} x^{a+1}(1-x)^{k-1} d x-\int_{0}^{y} x^{a+1}(1-x)^{k-1} d x\right) \\
& =(a+1-b+k) y^{b} \int_{y}^{1} x^{a+1}(1-x)^{k-1} d x .
\end{aligned}
$$

Similarly, for the third and fifth terms, we have

$$
\begin{gathered}
-(a+1-b-k) y^{a+1} \int_{0}^{y} x^{b}(1-x)^{k-1} d x+\frac{b !(k-1) !(a+1-b-k)}{(b+k) !} y^{a+1} \\
=(a+1-b-k) y^{a+1} \int_{y}^{1} x^{b}(1-x)^{k-1} d x .
\end{gathered}
$$

Therefore, (22) becomes

$$
\begin{aligned}
\tau(\mu, k, t)=\left(\begin{array}{c}
n \\
k-1, a+1, b
\end{array}\right) \cdot e^{-t(a b+b)}\left[\frac{(a+1-b) y^{a+b+2}(1-y)^{k-1}}{k}\right. \\
+y^{b}(a+1-b+k) \int_{y}^{1} x^{a+1}(1-x)^{k-1} d x \\
\left.+y^{a+1}(a+1-b-k) \int_{y}^{1} x^{b}(1-x)^{k-1} d x\right]=\phi(\mu, k, t)
\end{aligned}
$$

as desired.

Finally, let us treat the case $b=0$, in which $\mu=[n-k]$. We will see that this case is covered by the calculation in [7]. By [3, lemma 3],

$$
\operatorname{ch}\left(\alpha_{k}\right)=\frac{1}{k} u_{k} S_{[n-k]}
$$


by (11),

$$
\operatorname{ch}\left(\alpha_{k}\right)=\frac{1}{k} \sum_{\lambda \in E_{k}(\mu)}(-1)^{\mathrm{ht}(\lambda \backslash \mu)+1} S_{\lambda}
$$

which is equivalent to

$$
\alpha_{k}=\frac{1}{k} \sum_{\lambda \in E_{k}(\mu)}(-1)^{\mathrm{ht}(\lambda \backslash \mu)+1} \chi_{\lambda} .
$$

By lemma 5,

$$
\begin{aligned}
\mathbb{E}\left(\alpha_{k}(\pi(t))\right) & =\frac{1}{k} \sum_{\lambda \in E_{k}(\mu)}(-1)^{h t(\lambda \backslash \mu)+1} d_{\lambda} e^{-t \rho(\lambda)} \\
& =\frac{1}{k} \tau(\mu, k, t)
\end{aligned}
$$

On the other hand, $\mathbb{E}\left(\alpha_{k}(\pi(t))\right.$ has been calculated in [7, Theorem 1]:

$$
\mathbb{E}\left(\alpha_{k}(\pi(t))\right)=\left(\begin{array}{l}
n \\
k
\end{array}\right)\left[\frac{1}{k} y^{n-k+1}(1-y)^{k-1}+\int_{y}^{1} x^{n-k}(1-x)^{k-1} d x\right]
$$

comparing the two formulas for $\mathbb{E}\left(\alpha_{k}(\pi(t))\right)$, we conclude that $\tau(\mu, k, t)=\phi(\mu, k, t)$ for $b=0$ as well.

Proof of theorem 2. Theorem 2 is a direct consequence of theorem 17, the definition of $m^{2}(t)$ and $Z$ (equation (1)), the fact that $\psi(b, k)=(a+1-b) \phi([n-k-b, b], k, t)$ ( $\psi$ from theorem 2 and $\phi$ from theorem 17) and the fact that $\sum k \alpha_{k}=n$ so $Z=\mathbb{E} 2^{\alpha}=\frac{1}{n} \sum k \mathbb{E}\left(\alpha_{k} 2^{\alpha}\right)$.

\section{Proof of TheOREM 1}

The proof of theorem 1 is nothing but estimating carefully the terms in theorem 2 and is pretty straightforward, but let us anyway start with a bird's eye view. Recall the terms $\psi(b, k, t)$ from theorem 2 and that

$$
m_{n}^{2}(t)=n \frac{\sum_{b, k} k \psi(b, k, t)}{\sum_{b, k} \psi(b, k, t)} .
$$

where the sums are over all ranges of the parameters i.e. $k$ runs from 1 to $n$ and $b$ runs from 0 to $\lfloor(n-k) / 2\rfloor$. We will find the set of $(b, k)$ for which $\psi$ is maximal (depending on $t$ ) and the rest will turn out to be negligible. For visualisation purposes it is convenient to define normalised values $\tau=t n, \kappa=k / n$ and $\beta=b / n$ (no relation to the $\beta$ from $\S 1.4$ or the $\tau$ from $\S 6$ ). The relation $b \leq \frac{1}{2}(n-k)$ translates to $\beta \leq \frac{1}{2}(1-\kappa)$ (see the diagonal in figure 4 ). We return therefore to the definition of $\psi$ in theorem 2 and recall that it has three terms (below we abbreviate $\psi(b, k)=\psi(b, k, t))$. It will turn out that the term containing $\int_{y}^{1} x^{a+1}(1-x)^{k-1}$ (recall that $a=n-k-b$, that $a \geq b$ and that $y=e^{-t k}$ ) is the most significant one. The integrand has its maximum in the interval $[0,1]$ at $(a+1) /(a+k)$ and, as we will see, cases where the integral does not capture this maximum, i.e. that $y>(a+1) /(a+k)$, are also negligible. Thus we may restrict our attention to the corresponding part of the $\kappa, \beta$ plane. Since $y=e^{-\tau \kappa}$ and

$$
\frac{a+1}{a+k}=\frac{n-k-b+1}{n-b}=\frac{1-\kappa-\beta}{1-\beta}+o(1)
$$




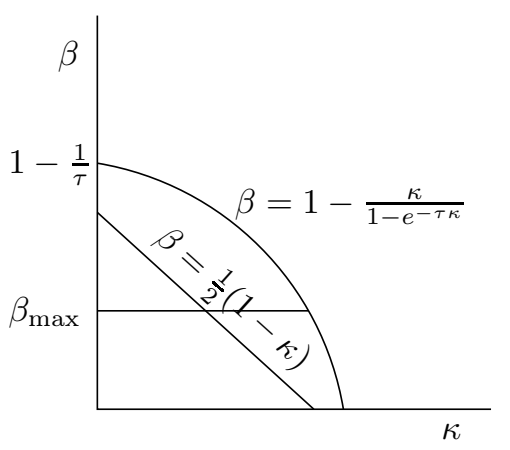

Figure $4 . \quad \beta$ and $\tau$ (the case $\tau>2$ depicted)

the condition becomes $\beta \leq 1-\kappa /\left(1-e^{-\tau \kappa}\right)$ (see figure 4 , and especially note the intersection with the $\kappa=0$ line, $\beta=1-1 / \tau)$. Further, when the integral does capture the maximum, it may be estimated very well by the integral from 0 to 1 , which is a Beta integral and has an explicit formula. We get for such $b$ and $k$,

$$
\begin{array}{r}
\psi(b, k) \sim(a+1-b)\left(\begin{array}{c}
n \\
k-1, a+1, b
\end{array}\right) e^{-t(a b+b)} y^{b}(a+1-b+k) \int_{0}^{1} x^{a+1}(1-x)^{k-1} \\
=\left(\begin{array}{c}
n \\
b
\end{array}\right) e^{-t b(n-b+1)}(n-k-2 b+1) \frac{n+1-2 b}{n-k} .
\end{array}
$$

The right-hand side depends rather weakly on $k$. Dropping some polynomial terms (in particular all terms containing $k$ ) we may write

$$
\begin{aligned}
\psi(b, k) & \sim\left(\begin{array}{l}
n \\
b
\end{array}\right) e^{-t b(n-b)} \\
& \sim\left(\frac{n}{b}\right)^{b}\left(\frac{n}{n-b}\right)^{n-b} e^{-t b(n-b)}=\left(\beta^{-\beta}(1-\beta)^{-(1-\beta)} e^{-\tau \beta(1-\beta)}\right)^{n}
\end{aligned}
$$

We see that, as a function of $\beta$, its maximum can be found by differentiating the expression on the right, and then solving a transcendental equation. Denote the $\beta$ at which the maximum is attained by $\beta_{\max }$. We do not care about the exact value of $\beta_{\max }$, we only need to know whether the line $\left\{\beta=\beta_{\max }\right\}$ intersects the region below both the curve and the diagonal in figure 4 (they are not always arranged as in the picture, this depends on the value of $\tau$ ), and for this we need only check the points $(\beta \in\{1-1 / \tau, 1 / 2\}, \kappa=0)$. A simple check shows that $\beta_{\max }<\min \left(\frac{1}{2}, 1-\frac{1}{\tau}\right)$ happens exactly when $\tau>2$, and hence this is the critical value for the appearance of large cycles: when $\tau<2$ the "mass" sits at the point $(\beta=\max (0,1-1 / \tau), \kappa=0)$ and in particular there are no large cycles. When $\tau>2$ there is approximately equal mass at each $k$ for which $\beta_{\max } \leq 1-\kappa /\left(1-e^{-\tau \kappa}\right)$ and in particular there are macroscopic cycles (very small $k$, of constant order, have larger mass, reflecting the fact that a positive proportion of the mass still sits in small cycles). The details of the calculation are below.

Proof. We start with the case of $\tau>2$ for which we need to show $m \gtrsim n$. Fix some $\tau>2$. Let $\mu$ be the function $\mu(\beta)=(1-\beta) \log (1-\beta)+\beta \log \beta+\tau \beta(1-\beta)$, let $\beta_{\max }$ be the unique minimiser of $\mu$ in the interval $\left[0, \frac{1}{2}\right]$, and note that $\beta_{\max }<\frac{1}{2}$ (here 
and below we skip the details of the calculus exercises involved). Let $\kappa_{0}>0$ satisfy $\beta_{\max }<1-\kappa_{0} /\left(1-e^{-\tau \kappa_{0}}\right)$ (note that $1-\kappa /\left(1-e^{-\tau \kappa}\right)$ is a decreasing function of $\kappa$, so that the inequality $\beta_{\max }<1-\kappa /\left(1-e^{-\tau \kappa}\right)$ holds for all $\left.\kappa<\kappa_{0}\right)$. Assume in addition that $\kappa_{0}<\frac{1}{2}-\beta_{\max }$. We will estimate, for $k<\kappa_{0} n$, the contribution of the term $\sum_{b} k \psi(b, k) / \sum_{l, b} \psi(b, l)$ at time $t=\tau / n$ and this will establish the theorem in the case $\tau>2$. Hence we need to bound $\sum_{b, l} \psi(b, l)$ from above and $\sum_{b} \psi(b, k)$ from below.

For the lower bound we first note that $\psi(b, k) \geq 0$ for all $b$ and $k$. Indeed, recall that the definition of $\psi$ (page 4 ) has three summands, two (the second and third) involving partial beta integrals and one not involving any integral. The only summand which might be negative is the third, because of the term $a+1-b-k$ which is sometimes negative (recall that $a=n-k-b$ and $y=e^{-t k}$ ). But the second summand cancels it. Indeed, because $a+1>b$,

$$
y^{a+1} \int_{y}^{1} x^{b}(1-x)^{k-1} \leq y^{b} \int_{y}^{1} x^{a+1}(1-x)^{k-1}
$$

and the sum of the two integrals in the definition of $\psi$ is bigger or equal to $2(a+$ $1-b) y^{b} \int_{y}^{1} x^{a+1}(1-x)^{k-1}$ and in particular is positive.

Therefore, to prove a lower bound we may restrict our attention to only a subset of the allowed $b$ and $k$. We take $b$ such that $\left|b-n \beta_{\max }\right| \leq 2 \sqrt{n}$ and $k \in\left(\frac{1}{2} n \kappa_{0}, n \kappa_{0}\right)$. Next we drop the first and third summands in the definition of $\psi$, which we may as they are positive (the term $a+1-b-k$ is positive for our values of $b$ and $k$ because of our assumption that $\kappa_{0}<\frac{1}{2}-\beta_{\max }$, if $n$ is sufficiently large). We get

$\psi(b, k) \geq(a+1-b) \frac{n !}{(k-1) !(a+1) ! b !} e^{-t(a b+b)} y^{b}(a+1-b+k) \int_{y}^{1} x^{a+1}(1-x)^{k-1} d x$.

Since $\beta_{\max }<\frac{1}{2}-\kappa_{0}$ we have

$$
a+1-b \gtrsim n
$$

(here and throughout the proof, the implicit constant in the notation $\gtrsim$ may depend only on $\tau$ and $\kappa_{0}$ ). Further, we claim that

$$
\int_{y}^{1} x^{a+1}(1-x)^{k-1} d x \gtrsim \int_{0}^{1} x^{a+1}(1-x)^{k-1} .
$$

To see (28) we first compare the integral to the maximum of its integrand. On the one hand, the integral from 0 to 1 is equal to $(k-1) !(a+1) ! /(a+k+1)$ !. On the other hand the maximum of the integrand is at $x_{\max }=(a+1) /(a+k)$. With Stirling's formula we get

$$
\int_{0}^{1} x^{a+1}(1-x)^{k-1} \approx \frac{\sqrt{k}}{n} x_{\max }^{a+1}\left(1-x_{\max }\right)^{k-1}
$$

where the notation $\approx$ means that the ratio between the two sides is bounded above and below by constants depending only on $\tau$ and $\kappa_{0}$ (the estimate (29) holds for any $b$ and $k$, not just under the restrictions above).

Returning to (28), we note that

$$
x_{\max }=1-\frac{k-1}{n-b}=1-\frac{\kappa}{1-\beta_{\max }}+O\left(n^{-1 / 2}\right) .
$$


Our choice of parameters gives $e^{-\tau \kappa}<1-\kappa /\left(1-\beta_{\max }\right)$, and this holds uniformly in $\kappa$, i.e.

$$
y=e^{-\tau \kappa}<1-\frac{\kappa}{1-\beta_{\max }}+c\left(\tau, \kappa_{0}\right)=x_{\max }+c\left(\tau, \kappa_{0}\right)+O\left(n^{-1 / 2}\right)
$$

for some $c\left(\tau, \kappa_{0}\right)>0$. Returning to $x^{a+1}(1-x)^{k-1}$ it is easy to check that it decays exponentially away from its maximum. All these give us that

$$
\int_{0}^{y} x^{a+1}(1-x)^{k-1} \leq y^{a+1}(1-y)^{k-1}<\left(1-c_{c}\left(\tau, \kappa_{0}\right)\right)^{n} x_{\max }^{a+1}\left(1-x_{\max }\right)^{k-1} .
$$

The exponential factor $\left(1-c_{2}\right)^{n}$ wins over the factor $\sqrt{k} / n$ in (29), so we get $\int_{0}^{y} \ll \int_{0}^{1}$. This establishes (28) for $n$ sufficiently large, and hence for every $n$ (perhaps with a different value for the constant implicit in the notation $\gtrsim$ ).

We get

$$
\begin{aligned}
\psi(b, k) & \geq(a+1-b) \frac{n !}{(k-1) !(a+1) ! b !} e^{-t(a b+b)} y^{b}(a+1-b+k) \cdot \\
& \quad \int_{y}^{1} x^{a+1}(1-x)^{k-1} d x \\
& \gtrsim n \frac{n !}{(k-1) !(a+1) ! b !} e^{-t b(a+1)} e^{-t k b} \cdot n \cdot \int_{0}^{1} x^{a+1}(1-x)^{k-1} d x \\
& =n^{2} \frac{n !}{(k-1) !(a+1) ! b !} e^{-t b(a+1+k)} \frac{(a+1) !(k-1) !}{(a+k+1) !} \\
& =n^{2} \frac{n !}{b !(n-b+1) !} e^{-t b(n-b+1)} \gtrsim n\left(\begin{array}{c}
n \\
b
\end{array}\right) e^{-t b(n-b)}
\end{aligned}
$$

where in the last inequality we used that $e^{-t b} \approx 1$ and that $n-b \approx n$. This holds for all $b$ satisfying $\left|b-n \beta_{\max }\right| \leq 2 \sqrt{n}$, and for such $b$ we have, by Stirling's formula,

$$
\begin{aligned}
\left(\begin{array}{l}
n \\
b
\end{array}\right) e^{-t b(n-b)} & \approx \frac{1}{\sqrt{n}} \exp \left(-\left(b \log \frac{b}{n}+(n-b) \log \frac{n-b}{n}+t b(n-b)\right)\right) \\
& =\frac{1}{\sqrt{n}} \exp \left(-n \mu\left(\frac{b}{n}\right)\right) \gtrsim \frac{1}{\sqrt{n}} \exp \left(-n \mu\left(\beta_{\max }\right)\right)
\end{aligned}
$$

where the second inequality comes from Taylor expanding $\mu$ near $\beta_{\max }$ to second order (since $\beta_{\max }$ is the minimum of $\mu, \mu^{\prime}\left(\beta_{\max }\right)=0$ ). Summing over $b$ thus gives

$$
\sum_{b} \psi(b, k) \gtrsim n \exp \left(-n \mu\left(\beta_{\max }\right)\right) \text {. }
$$

This ends the lower bound.

For the upper bound we need to consider all 3 summands in the definition of $\psi$ in theorem 2, as well as the case $b=0$. The easiest are the integrals (the second and third summands). For the second we write

$$
y^{b} \int_{y}^{1} x^{a+1}(1-x)^{k-1} d x<y^{b} \int_{0}^{1} x^{a+1}(1-x)^{k-1} d x .
$$

For the third, (26) gives the same estimate:

$$
y^{a+1} \int_{y}^{1} x^{b}(1-x)^{k-1} d x \stackrel{(26,32)}{<} y^{b} \int_{0}^{1} x^{a+1}(1-x)^{k-1} .
$$


For the first summand we use (29) to write

$$
y^{a+b+2}(1-y)^{k-1} \leq x_{\max }^{a+1}\left(1-x_{\max }\right)^{k-1} y^{b+1} \stackrel{(29)}{\lesssim} \frac{n}{\sqrt{k}} y^{b} \int_{0}^{1} x^{a+1}(1-x)^{k-1} .
$$

(the divergence as $k \rightarrow 1$ reflects the fact that indeed, in our time scale, there are still many small cycles). We covered the three terms in theorem 2 in (32), (33) and (34) so we may write

$$
\begin{aligned}
\psi(b, k, t) & \lesssim\left(\begin{array}{c}
n \\
k-1, a+1, b
\end{array}\right) e^{-t b(a+1)} \cdot n^{2}\left(1+n k^{-3 / 2}\right) y^{b} \int_{0}^{1} x^{a+1}(1-x)^{k-1} \\
& =\frac{n !}{(n-b+1) ! b !} n^{2}\left(1+n k^{-3 / 2}\right) e^{-t b(n-b-1)} \\
& \lesssim n\left(\begin{array}{c}
n \\
b
\end{array}\right) e^{-t b(n-b)}\left(1+n k^{-3 / 2}\right) .
\end{aligned}
$$

Formally, we only showed (35) for $b>0$, but an inspection of the case $b=0$ shows that it differs from that $b>0$ case only in details of the polynomial factors and in terms such as $y^{C}$, all of which may be "folded" into the implicit constant. Hence (35) holds also for $b=0$.

All that remains is to sum (35) over all $k$ and $b$. Summing over $k$ simply gives another factor of $n$ i.e. $n^{2}\left(\begin{array}{l}n \\ b\end{array}\right) e^{-t b(n-b)}$. As for the sum over $b$, a simple calculus exercise shows that our function $\mu$ defined by $\mu(\beta)=\beta \log \beta+(1-\beta) \log (1-\beta)+$ $\tau \beta(1-\beta)$ satisfies

$$
\mu(\beta) \geq \mu\left(\beta_{\max }\right)+c\left(\beta-\beta_{\max }\right)^{2}
$$

for some constant $c$ depending only on $\tau$. Hence we have

$$
\begin{aligned}
& \sum_{b=0}^{n / 2}\left(\begin{array}{l}
n \\
b
\end{array}\right) e^{-t b(n-b)} \lesssim \sum_{b=0}^{n / 2} \frac{1}{\sqrt{n}} \exp \left(-n \mu\left(\frac{b}{n}\right)\right) \\
& \quad \lesssim \frac{1}{\sqrt{n}} \exp \left(-n \mu\left(\beta_{\max }\right)\right) \sum_{b=0}^{n / 2} \exp \left(-n c\left(\frac{b}{n}-\beta_{\max }\right)^{2}\right) \lesssim \exp \left(-n \mu\left(\beta_{\max }\right)\right)
\end{aligned}
$$

(we skip the details of the last calculation, which is standard). All in all we get

$$
\sum_{k, b} \psi(b, k) \lesssim n^{2} \exp \left(-n \mu\left(\beta_{\max }\right)\right) .
$$

With (31) the case of $\tau>2$ is finished: we get, for every $k \in\left(\frac{1}{2} n \kappa_{0}, n \kappa_{0}\right)$

$$
\frac{\sum_{b} \psi(b, k)}{\sum_{b, l} \psi(b, l)} \gtrsim \frac{n \exp \left(-n \mu\left(\beta_{\max }\right)\right)}{n^{2} \exp \left(-n \mu\left(\beta_{\max }\right)\right)}=\frac{1}{n} .
$$

Hence

$$
m^{2}=n \sum_{k=0}^{n} k \frac{\sum_{b} \psi(b, k)}{\sum_{b, l} \psi(b, l)} \gtrsim n \sum_{k=\left\lceil\frac{1}{2} n \kappa_{0}\right\rceil}^{\left\lfloor n \kappa_{0}\right\rfloor} \frac{k}{n} \gtrsim n^{2}
$$

as needed. 
The case $\tau<2$. We retain the notation $\mu(\beta)=\beta \log \beta+(1-\beta) \log (1-\beta)+\tau \beta(1-$ $\beta$ ) from the previous part, but note that in this case this function is decreasing on all of $\left[0, \frac{1}{2}\right]$. Again we need upper and lower bounds on $\psi(b, k)$.

The lower bound follows by considering $k=1$ and $b \in\left[\frac{1}{2} n-2 \sqrt{n}, \frac{1}{2} n-\sqrt{n}\right]$, and by dropping the two terms in the definition of $\psi$ which contain integrals. (Recall that $\psi(b, k) \geq 0$ for all $b$ and $k$, which explains why we may consider only a subset of the $k$ and $b$, and that the sum of the two integral terms sum is positive. Both facts are explained in the discussion in the beginning of the proof of the case $\tau>2$, around formula (26)). This gives

$$
\psi(b, k) \geq(a-1+b)^{2}\left(\begin{array}{l}
n \\
b
\end{array}\right) e^{-t(a b+b)} y^{a+b+2} \gtrsim n \cdot \frac{2^{n}}{\sqrt{n}} \cdot e^{-n \tau / 4} .
$$

Since there are $\approx \sqrt{n}$ terms with this estimate we get

$$
\sum_{b, k} \psi(b, k) \gtrsim n 2^{n} e^{-n \tau / 4}
$$

Notice that the exponential terms are exactly $\exp \left(-n \mu\left(\frac{1}{2}\right)\right)$.

For the upper bound we need to split into two cases: $\beta=b / n<\frac{1}{2}-(2-\tau) / 10$ and the complement. We start with the first, for which we can use (35) as is (its proof did not use any assumptions on $\tau, b$ or $k$ ). We get

$$
\psi(b, k) \lesssim n^{2}\left(\begin{array}{l}
n \\
b
\end{array}\right) e^{-t b(n-b)} \lesssim n^{3 / 2} \exp (-n \mu(\beta))
$$

and because $\beta<\frac{1}{2}$ and $\mu$ is strictly decreasing, $\psi(b, k) \lesssim n 2^{n} e^{-n \tau / 4}(1-c)^{n}$ for some $c(\tau)>0$. Thus these terms are negligible.

The other case is $\beta \geq \frac{1}{2}-(2-\tau) / 10$. In this case

$$
e^{-\tau \kappa}>1-\frac{\kappa}{1-b / n}
$$

which means that for all $k$ sufficiently large, $y>x_{\max }+c(\tau) \kappa$ (recall that $x_{\max }=$ $1-(k-1) /(n-b))$. This gives an estimate for the integrals in the definition of $\psi$ by their value at $y$ times the length of the integration interval (up to a constant, for $k \lesssim 1$ ). For example,

$$
\begin{aligned}
\int_{y}^{1} x^{a+1}(1-x)^{k-1} & \lesssim(1-y) \cdot y^{a+1}(1-y)^{k-1} \lesssim \frac{k}{n} x_{\max }^{a+1}\left(1-x_{\max }\right)^{k-1}(1-c)^{k} \\
& \stackrel{(29)}{\lesssim} \frac{k}{n} \cdot \frac{n}{\sqrt{k}} \cdot \frac{(a+1) !(k-1) !}{(a+k+1) !}(1-c)^{k}
\end{aligned}
$$

A similar estimate holds for the other integral. The remaining summand in the definition of $\psi$ has a similar estimate, but without the length of the integration 
interval, i.e. the term $\frac{k}{n}$ above. We get

$$
\begin{aligned}
\psi(b, k) & \lesssim(a+1-b)\left(\begin{array}{c}
n \\
k-1, a+1, b
\end{array}\right) e^{-t b(a+1)} y^{b} \cdot \frac{n}{\sqrt{k}} \frac{(a+1) !(k-1) !}{(a+k+1) !}(1-c)^{k} . \\
& {\left[\frac{(a+1-b) y}{k}+\frac{k}{n}(a+1-b+k)+\frac{k}{n}|a+1-b-k|\right] } \\
& \lesssim(n+1-2 b)^{2}\left(\begin{array}{l}
n \\
b
\end{array}\right) e^{-t b(n-b)} \frac{1}{\sqrt{k}}(1-c)^{k}\left(\frac{1}{k}+\frac{k}{n}\right) \\
& \stackrel{(30)}{\lessgtr} \frac{1}{\sqrt{n}}(n+1-2 b)^{2} \exp (-n \mu(\beta))(1-c)^{k} .
\end{aligned}
$$

Finally, writing $\mu(\beta) \geq \mu\left(\frac{1}{2}\right)-c(1-2 \beta)^{2}$ (compare to (36)) we get

$$
\begin{aligned}
\sum_{b} \psi(b, k) & \lesssim \frac{1}{\sqrt{n}} 2^{n} e^{-\tau n / 4}(1-c)^{k} \sum_{b=0}^{n / 2}(n+1-2 b)^{2} \exp \left(-c(1-2 b / n)^{2}\right) \\
& \lesssim n 2^{n} e^{-n \tau / 4}(1-c)^{k} .
\end{aligned}
$$

where the first sum is only over $b$ such that $b / n \geq \frac{1}{2}-(2-\tau) / 10$, and of course such that $b \leq \frac{1}{2}(n-k)$. Again, we omit the details of the last inequality. Since the sum over the other values of $b$ is negligible, we see that (39) in fact holds (perhaps with a different value of $c$ ) even when the first sum is over all $b$. The proof is now finished: we write

$$
m^{2}=n \sum_{k=1}^{n} k \frac{\sum_{b} \psi(b, k)}{\sum_{b, l} \psi(b, l)} \stackrel{(38,39)}{\lessgtr} n \sum_{k=1}^{n} k(1-c)^{k} \lesssim n
$$

as needed.

\section{Appendix A. The SPontaneous and the Residual magnetisations}

The purpose of this appendix is to show that for $t$ such that the expected square magnetisation $m$ satisfies $m(t) \gtrsim n$ we also have that the residual magnetisation $m^{*}$ (recall its definition (8)) satisfies $m^{*}(t)>0$. Recall that $Z=\mathbb{E}\left(2^{\alpha}\right)$ and $m^{2}=(1 / Z) \mathbb{E}\left(\left(\sum k^{2} \alpha_{k}\right) 2^{\alpha}\right)$. Let $\varepsilon$ be some positive number and write

$$
\begin{aligned}
\sum_{k} k^{2} \alpha_{k} & =\sum_{k \geq \varepsilon n} k^{2} \alpha_{k}+\sum_{k<\varepsilon n} k^{2} \alpha_{k} \leq n\left(\sum_{k \geq \varepsilon n} k \alpha_{k}\right)+\varepsilon n \sum_{k<\varepsilon n} k \alpha_{k} \\
& =n\left(\sum_{k \geq \varepsilon n} k \alpha_{k}\right) \mathbb{1}\left\{\sum_{k \geq \varepsilon n} \alpha_{k}>0\right\}+\varepsilon n \sum_{k<\varepsilon n} k \alpha_{k} \\
& \leq n^{2} \mathbb{1}\left\{\sum_{k \geq \varepsilon n} \alpha_{k}>0\right\}+\varepsilon n^{2}
\end{aligned}
$$

where in the second inequality we used $\sum k \alpha_{k}=n$. Now multiply by $2^{\alpha}$ and take expectations and get

$$
\mathbb{E}\left(\mathbb{1}\left\{\sum_{k \geq \varepsilon n} \alpha_{k}>0\right\} 2^{\alpha}\right) \geq \frac{1}{n^{2}} Z m^{2}-\varepsilon Z
$$


Recall our assumption that $m>c n$ for some $c>0$. Choose $\varepsilon=\frac{1}{2} c^{2}$ and get

$$
\mathbb{E}\left(\mathbb{1}\left\{\sum_{k \geq \varepsilon n} \alpha_{k}>0\right\} 2^{\alpha}\right) \geq \frac{1}{2} c^{2} Z
$$

Now examine the definition of $m^{*}$. Fix some $M$ and let $n>M / \varepsilon$. Then

$$
\sum_{k=M+1}^{n} \frac{k}{n} \alpha_{k} \geq \sum_{k \geq \varepsilon n} \frac{k}{n} \alpha_{k} \geq \varepsilon \mathbb{1}\left\{\sum_{k \geq \varepsilon n} \alpha_{k}>0\right\} .
$$

Again multiplying by $2^{\alpha}$ and taking expectations gives

$$
\sum_{k=M+1}^{n} \frac{k}{n} \mathbb{E}\left(\alpha_{k} 2^{\alpha(\pi(\beta))}\right) \geq \varepsilon \mathbb{E}\left(\mathbb{1}\left\{\sum_{k \geq \varepsilon n} \alpha_{k}>0\right\} 2^{\alpha}\right) \geq \varepsilon \cdot \frac{1}{2} c^{2} Z=\varepsilon^{2} Z
$$

or

$$
\varliminf_{n \rightarrow \infty} \frac{1}{Z_{n}(\beta)} \sum_{k=M+1}^{n} \frac{k}{n} \mathbb{E}\left(\alpha_{k} 2^{\alpha(\pi(\beta))}\right) \geq \varepsilon^{2} .
$$

Since $\varepsilon$ did not depend on $M$, we get $m^{*} \geq \varepsilon^{2}$, as needed.

\section{REFERENCES}

[1] Radosław Adamczak, Michał Kotowski and Piotr Miłoś, Phase transition for the interchange and quantum Heisenberg models on the Hamming graph. Available at: arXiv: 1808.08902

[2] Michael Aizenman and Bruno Nachtergaele, Geometric aspects of quantum spin states. Comm. Math. Phys. 164 (1994), no. 1, 17-63. Available at: projecteuclid.org/1104270709

[3] Gil Alon and Gady Kozma, The probability of long cycles in interchange processes, Duke Math. J. 162:9 (2013), 1567-1585. Available at: projecteuclid.org/1370955539

[4] Gil Alon and Gady Kozma, Comparing with octopi. In preparations.

[5] Omer Angel, Random infinite permutations and the cyclic time random walk. Discrete random walks (Paris, 2003), 9-16, Discrete Math. Theor. Comput. Sci. Proc. conference volume AC. Available at: emis.ams/dmAC0101

[6] Nathanaël Berestycki and Rick Durrett, A phase transition in the random transposition random walk. Probab. Theory Related Fields 136:2 (2006), 203-233. Available at: springer. com/005-0479-7

[7] Nathanaël Berestycki and Gady Kozma, Cycle structure of the interchange process and representation theory, Bull. Soc. Math. France 143:2 (2015), 265-280. smf.ft/143_265-281

[8] Nathanaël Berestycki, Oded Schramm and Ofer Zeitouni, Mixing times for random $k$-cycles and coalescence-fragmentation chains. Ann. Probab. 39:5 (2011), 1815-1843. Available at: projecteuclid.org/1318940782

[9] Jakob E. Björnberg, Large cycles in random permutation related to the Heisenberg model. Electron. Commun. Probab. 20 (2015), paper 55, 11 pp. Available at: projecteuclid/ 1465320982

[10] Jakob E. Björnberg, The free energy in a class of quantum spin systems and interchange processes. J. Math. Phys. 57 (2016), 073303, 17 pp. Available at: aip.org/1.4959238

[11] Joseph G. Conlon and Jan Philip Solovej, Random walk representations of the Heisenberg model. J. Statist. Phys. 64:1-2 (1991), 251-270. Available at: springer.com/BF01057876

[12] Michele Correggi, Alessandro Giuliani and Robert Seiringer, Validity of the spin-wave approximation for the free energy of the Heisenberg ferromagnet. Comm. Math. Phys. 339:1 (2015), 279-307. Available at: springer.com/s00220-015-2402-0

[13] Nicholas Crawford and Dmitry Ioffe, Random current representation for transverse field Ising model. Comm. Math. Phys. 296:2 (2010), 447-474. springer.com/s00220-010-1018-7

[14] Persi Diaconis, Applications of noncommutative Fourier analysis to probability problems. École d'Été de Probabilités de Saint-Flour XV-XVII, 1985-87, 51-100, Lecture Notes in Math., 1362, Springer, Berlin, 1988. 
[15] Persi Diaconis and Laurent Saloff-Coste, Comparison techniques for random walk on finite groups. Ann. Probab. 21:4 (1993), 2131-2156. Available at: jstor.org/2244713

[16] Persi Diaconis and Mehrdad Shahshahani, Generating a random permutation with random transpositions. Z. Wahrsch. Verw. Gebiete 57:2 (1981), 159-179. springer.com/BF00535487

[17] Freeman J. Dyson, Elliott H. Lieb and Barry Simon, Phase transitions in quantum spin systems with isotropic and nonisotropic interactions. J. Statist. Phys. 18:4 (1978), 335-383. Available at: springer.com/BF01106729

[18] Georg F. Frobenius. Über die Charaktere der symmetrischen Gruppe. [German, On the characters of the symmetric group]. Sitzungsberichte der Königlich Preussischen Akademie der Wissenschaften zu Berlin 1900, 516-534. Available at: bibliothek.de/1900-1: int=530

[19] Jürg Fröhlich, Robert Israel, Elliott H. Lieb and Barry Simon, Phase transitions and reflection positivity. I. General theory and long range lattice models. Comm. Math. Phys. 62:1 (1978), 1-34. Available at: projecteuclid.org/1103904299

[20] Jürg Fröhlich, Robert Israel, Elliott H. Lieb and Barry Simon, Phase transitions and reflection positivity. II. Lattice systems with short-range and Coulomb interactions. J. Statist. Phys. 22:3 (1980), 297-347. Available at: springer.com/BF01014646

[21] Jürg Fröhlich, Barry Simon and Thomas Spencer, Infrared bounds, phase transitions and continuous symmetry breaking. Comm. Math. Phys. 50:1 (1976), 79-95. Available at: projecteuclid.org/1103900151

[22] Alan Hammond, Infinite cycles in the random stirring model on trees. Bull. Inst. Math. Acad. Sin. (N.S.) 8:1 (2013), 85-104.

[23] Alan Hammond, Sharp phase transition in the random stirring model on trees. Probab. Theory Related Fields 161:3-4 (2015), 429-448. Available at: springer.com/s00440-013-0543-7

[24] Gordon James and Adalbert Kerber, The representation theory of the symmetric group. Encyclopedia of Mathematics and its Applications, 16. Addison-Wesley Publishing Co., Reading, Mass., 1981.

[25] Roman Kotecký, Piotr Miłoś and Daniel Ueltschi, The random interchange process on the hypercube. Electron. Commun. Probab. 21 (2016), paper 4. projecteuclid.org/1454514624

[26] Oliver Penrose, Bose-Einstein condensation in an exactly soluble system of interacting particles, J. Statist. Phys. 63:3-4 (1991), 761-781. Available at: springer.com/BF01029210

[27] Robert T. Powers, Heisenberg model and a random walk on the permutation group. Lett. Math. Phys. 1:2 (1975/76), 125-130. Available at: springer.com/BF00398374

[28] Mercedes H. Rosas. The Kronecker product of Schur functions of two row shapes or hook shapes. J. Alg. Combin. 14:2 (2001), 153-173. springer.com/A:1011942029902

[29] Oded Schramm, Compositions of random transpositions. Israel J. Math. 147 (2005), 221-243. Available at: springer.com/BF02785366

[30] Frank Spitzer, Interaction of Markov processes. Advances in Math. 5 (1970), 246-290. Available at: sciencedirect.com/900344

[31] Wolfgang Spitzer, Shannon Starr and Lam Tran, Counterexamples to ferromagnetic ordering of energy levels. J. Math. Phys. 53:4 (2012), 043302. Available at: aip.org/1.3699015

[32] Richard P. Stanley, Enumerative Combinatorics, volume 1. Cambridge University Press, 1997.

[33] Richard P. Stanley, Enumerative Combinatorics, volume 2. Cambridge University Press, 1999.

[34] Bálint Tóth, Phase transition in an interacting Bose system. An application of the theory of Ventsel' and Freı̆dlin. J. Statist. Phys. 61:3-4 (1990), 749-764. springer.com/BF01027300

[35] Bálint Tóth, Improved lower bound on the thermodynamic pressure of the spin 1/2 Heisenberg ferromagnet. Lett. Math. Phys. 28:1 (1993), 75-84. Available at: springer.com/BF00739568

[36] Natalia Tsilevich, Spectral properties of the periodic Coxeter Laplacian in the two-row ferromagnetic case. PDMI preprint 02/2010, available from: pdmi.ru/ natalia

[37] Daniel Ueltschi, Random loop representations for quantum spin systems. J. Math. Phys. 54:8 (2013), 083301. Available at: aip.org/1.4817865

[38] Alfred Young, Quantitative substitutional analysis II, Proc. London Math. Sot., Ser. 1, 35 (1902), 361-397.

GA: Department of Mathematics and Computer Science, The Open University of IsRAEL, 4353701 RAANANA, IsRAEL

GK: Department of Mathematics and Computer Science, The Weizmann Institute of Science, 76100 Rehovot, Israel. 